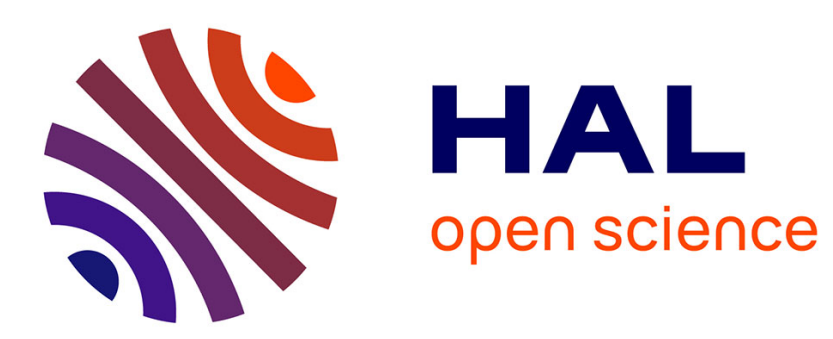

\title{
Pricing Ratchet equity-indexed annuities with early surrender risk in a CIR ++ model
}

\author{
Xiao Wei, Marcellino Gaudenzi, Antonino Zanette
}

\section{To cite this version:}

Xiao Wei, Marcellino Gaudenzi, Antonino Zanette. Pricing Ratchet equity-indexed annuities with early surrender risk in a CIR++ model. North American Actuarial Journal, 2013, 17 (3), pp.229-252. 10.1080/10920277.2013.826126 . hal-00721963

\section{HAL Id: hal-00721963 https://hal.inria.fr/hal-00721963}

Submitted on 31 Jul 2012

HAL is a multi-disciplinary open access archive for the deposit and dissemination of scientific research documents, whether they are published or not. The documents may come from teaching and research institutions in France or abroad, or from public or private research centers.
L'archive ouverte pluridisciplinaire HAL, est destinée au dépôt et à la diffusion de documents scientifiques de niveau recherche, publiés ou non, émanant des établissements d'enseignement et de recherche français ou étrangers, des laboratoires publics ou privés. 
Pricing Ratchet equity-indexed annuities with early surrender risk in a CIR ++ model

Xiao Wei , Marcellino Gaudenzi, Antonino Zanette

\section{RESEARCH}

REPORT

$\mathbf{N}^{\circ} 8034$

July 31, 2012

Project-Team Mathrisk 



\title{
inzián
}

\section{Pricing Ratchet equity-indexed annuities with early surrender risk in a CIR ++ model}

\author{
Xiao Wei *, Marcellino Gaudenzi ${ }^{\dagger}$, Antonino Zanette ${ }^{\dagger}$ \\ Project-Team Mathrisk
}

Research Report n 8034 - July 31, 2012 - 26 pages

\begin{abstract}
In connection with a problem posed by Kijima and Wong [11], we propose a lattice algorithm for pricing simple Ratchet equity-indexed annuities (EIAs) with early surrender risk and global minimum contract value when the asset value depends on the $\mathrm{CIR}++$ stochastic interest rates. In addition we present an asymptotic expansion technique which permits to obtain a first order approximation formula for the price of simple Ratchet EIAs without early surrender risk and without global minimum contract value. Numerical comparisons show the reliability of the proposed methods.
\end{abstract}

Key-words: Ratchet Equity-indexed annuities; stochastic interest rates; early surrender risk; lattice methods; asymptotic expansions

This research was supported by MIUR (Prin 2007) and by NSFC (No. 10801139)

* China Institute for Actuarial Science, Central University of Finance and Economics, Beijing, China, weix@cias.edu.cn

$\dagger$ Dipartimento di Scienze Economiche e Statistiche, Via Tomadini 30/A, Università di Udine, 33100 Udine, Italy (E-mail: gaudenzi@uniud.it, antonino.zanette@uniud.it)

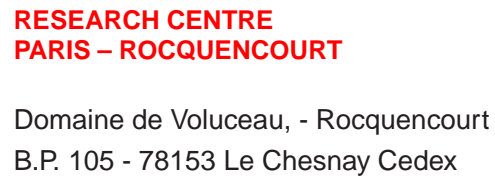




\section{Pricing de Ratchet sur action avec risque de rachat anticipé et utilisant le modèle CIR ++}

Résumé : Suite au problème posé par Kijima et Wong, nous proposons un algorithme de treillis pour le pricing du Ratchet sur action avec risque de rachat anticipé. Il inclut aussi une valeur minimale globale lorsque l'actif dépend du processus CIR ++ du taux d'intérêt. Par ailleurs, nous présentons une technique de développement asymptotique permettant une approximation de premier ordre pour le prix du Ratchet EIAs, sans risque de rachat anticipé ni valeur minimale. Des expériences numériques montrent une bonne qualité des résultats obtenus par la méthode proposée.

Mots-clés : Ratchet sur action, taux d'intérêt stochastiques, méthode de treillis, risque de rachat anticipé, développement asymptotique 


\section{Introduction}

Equity Indexed Annuities (EIAs) are important contracts in the insurance market. Ratchet EIAs allow the policyholder to participate in the potential appreciation of the stock market. Moreover, in order to eliminate the downside risk, the insurance companies typically guarantee a minimum return. EIAs were introduced in 1995 by Keyport Life Insurance Co., and now appeal more and more investors. In the early beginning, EIAs were priced in the framework of the standard Black-Scholes model, that is, the equity index follows a geometric Brownian motion process with a constant interest rate (see Gerber and Shiu [8], Hardy [9]). But EIAs usually have a long maturity, hence it's more preferable to price EIAs under a stochastic interest rates model rather than under a constant one. Up to now, Vasicek model is the only stochastic interest rates model used in EIAs pricing as we know. Lin and Tan ([15]) obtained prices for various types of EIAs, in the Vasicek model, by using Monte Carlo simulations. Kijima and Wong ([11]) derived a closed formula, in the extended Vasicek interest rates model, for pricing simple Ratchet EIAs with geometric index averaging. They also propose an efficient Monte Carlo simulation algorithm for pricing Ratchet EIAs with arithmetic index averaging. As well known, the main problem of the extended Vasicek model is that it permits negative interest rates. Moreover, as noted by Kijima and Wong, Ratchet EIAs pricing literature has largely ignored early surrender risk, so that they propose this question as a challenging problem.

In this paper we consider a joint evolution for the equity value with the CIR ++ stochastic interest rates model (see Brigo and Mercurio [3]) which is consistent with the term structure of the interest rates and it could overcome the problem of negative interest rates of the extended Vasicek model (see The Positivity of Rates and Fitting Quality section 3.9.3 of Brigo and Mercurio [3]). The purpose is two-fold: to obtain analytical approximations and to provide lattice techniques in order to treat early surrender risk.

We propose an asymptotic expansion formula, based on the approaches of Kim and Kunitomo [12], Kunitomo and Takahashi [13], for pricing simple Ratchet EIAs without minimum contract value and without early surrender risk.

Furthermore we introduce a second technique, a tree method which allows to price simple Ratchet EIAs with early surrender risk and with minimum contract value. In this case, the pricing problem leads to a non-recombining tree. In order to treat the problem we use the framework of singular points techniques introduced in Gaudenzi, Lepellere and Zanette [7] (see also Costabile, Gaudenzi, Massabò and Zanette [4]). The proposed algorithm is based on appropriate jump conditions at each node corresponding to each reset date. We obtain a procedure which allows to get the price in a reasonable time and to obtain easily the convergence of the discrete approximations to the continuous value.

The paper is organized as follows. In Section 2 we describe the financial model for the joint evolution of the equity and of the spot interest rate processes in a continuous setting. In Section 3 we describe the insurance contracts. In Section 4 the approximation of the price of simple Ratchet EIAs without early surrender risk and minimum contract value is obtained. In Section 5 we propose our tree algorithm for evaluating the price of the simple Ratchet EIAs with early surrender risk. Numerical results are proposed in Section 6.

\section{The financial model}

We shall be concerned with a geometric Brownian motion describing the evolution of the equity value with stochastic interest rates. We consider, under the risk-neutral probability measure $\mathbb{Q}$, 
the following dynamics for the equity value

$$
\frac{d S(t)}{S(t)}=r(t) d t+\sigma_{S} d B_{1}(t), \quad S(0)=S_{0}>0
$$

where $r(t)$ is the short interest rate, $\sigma_{S}$ is the constant stock price volatility and $B_{1}(t)$ is a standard Brownian motion.

The risk-neutralized process for the short rate $r(t)$ is described, as in the extended CoxIngersoll-Ross model $(\mathrm{CIR}++$ ) (see [5] and [3]) by the following dynamics:

$$
\left\{\begin{array}{l}
d x(t)=k(\theta-x(t)) d t+\sigma \sqrt{x(t)} d B_{2}(t), \quad x(0)=x_{0} \\
r(t)=x(t)+\varphi(t)
\end{array}\right.
$$

where $k$ is a constant representing the reversion speed, $\theta$ is the long term reversion target, $\sigma$ is a constant and $B_{2}(t)$ is a standard Brownian motion whose correlation with $B_{1}(t)$ is $\rho$. The Novikov condition

$$
2 k \theta>\sigma^{2}
$$

ensures that the origin is inaccessible to the process $x$, so that it remains positive. The function $\varphi$ is a deterministic function completely determined by the market values of the zero-coupon bonds (see [3]).

\section{The simple Ratchet EIAs with minimum contract value and early surrender risk}

We consider a simple Ratchet EAIs contract with annual reset dates and maturity $N$ years, whose payoff depends on the returns of the underlying asset at given reset times $1, \ldots, N$.

Let us denote by

$$
R_{i}=\frac{S(i)}{S(i-1)}, \quad i=1, \ldots, N,
$$

the return during the annual period $[i-1, i]$ of the underlying asset $S(i), i=0,1, \ldots, N$, at the annual observation times.

During every period, a local cap and floor of the individual returns, denoted by $C$ and $F$ respectively, will be taken into account.

The sum $Z_{i}$ defined by

$$
Z_{i}=1+\sum_{j=1}^{i} \max \left(F, \min \left(C, \alpha\left[R_{j}-1\right]\right)\right)
$$

will be called the running sum at time $i\left(Z_{0}=1\right)$. Here $\alpha>0$ is the participation rate. We name $\max \left(F, \min \left(C, \alpha\left[R_{j}-1\right]\right)\right)$ the Ratchet interest rate in the time period $[j-1, j]$.

Moreover a minimum contract value $(\mathrm{MCV})$

$$
G_{N}=\beta(1+g)^{N}
$$

is considered. Here $g \geq 0$ is the minimum guaranteed annual interest rate and $\beta$ is a certain percentage of the initial premium. This value can be considered as a global floor of the simple Ratchet contract. Hence $Z_{N}$ cannot be smaller than the global floor $G_{N}$. 
The payoff $\phi$ of the simple Ratchet EIAs with MCV is given by

$$
\phi\left(G_{N}, Z_{N}\right)=\max \left(G_{N}, Z_{N}\right) .
$$

For the simple Ratchet EIAs without MCV, the payoff function reduces to $Z_{N}$.

A surrender early risk can be embedded into the policy and it allows the policyholder to escape out of the contract at the beginning of each year. We consider the decision to surrender the policy as endogenous to the evaluation model, i.e., the policyholder decides to abandon the contract if this is financially convenient. This allows to treat the surrender case as a Bermudan option embedded into the simple Ratchet contract.

Let

$$
G_{i}=\beta(1+g)^{i}, \quad i=1, . ., N
$$

the minimum contact value at annual time $i$. At every reset date $i>0, i=1, \ldots, N-1$, the policyholder has two alternatives: to continue the simple Ratchet contract or to surrender it receiving the maximum between the running sum $Z_{i}$ and minimum contract value at time $i$

$$
\phi\left(Z_{i}, G_{i}\right)=\max \left(Z_{i}, G_{i}\right) .
$$

In the insurance practice cancellation of a contract in the early years may result in surrender charges. According to 2009 Annuity Fact Book ([20]), these charges generally consist in a given percentage of the annuity value and usually decline to zero over a period of time. The number of years for which the cancellation fee is applied will be denoted by $N_{c}\left(N_{c}<N\right)$. We shall denote by $\xi\left(i, N_{c}\right)$ the multiplicative factor needed in order to consider the cancellation charge. Hence surrendering the simple Ratchet contract the policyholder receives the amount (see [17]):

$$
\phi\left(Z_{i}, G_{i}\right)=\max \left(\xi\left(i, N_{c}\right) Z_{i}, G_{i}\right) .
$$

In the numerical examples we will choose the function $\xi$ according to 2009 Annuity Fact Book.

The evaluation of the previous contract requires the pricing of a Bermudan path-dependent contingent claim in the continuous bivariate model with the interest rate that follows the CIR ++ model.

\section{Asymptotic expansion method for EIAs without early sur- render risk and without $\mathrm{MCV}$}

In this section, we propose an expansion method for pricing simple Ratchet EIAs without early surrender risk and without MCV. This expansion, called small disturbance asymptotics, was already applied to price plain vanilla European options under a CIR stochastic interest rates model (see [13]). This approach permits to evaluate plain vanilla European financial contingent claims when the underlying asset prices follow a general class of continuous Itô processes. The rigorous mathematical validity of this expansion method was discussed in [13]. We propose here an expansion method for the simple Ratchet EIAs without MCV. In fact, the presence of the MCV causes difficulties in order to obtain an expansion because of the additional maximum operator in the payoff function (4).

In order to price EIAs without MCV in the extended $\mathrm{CIR}++$ model we consider first the asymptotic expansion of $\mathrm{CIR}++$ stochastic interest rate $r(t)$ around the interest rate volatility $\sigma=0$ using the Watanabe-Yoshida theory based on Malliavin Calculus (see [13]). Then we substitute the asymptotic expansion of the interest rate term in the expression of the return of the equity index. We finally derive a first order asymptotic expansion for the price of simple Ratchet EIAs from the previous computations. 
The price $V_{0}$ at time 0 of an $N$-year simple Ratchet EIAs without early surrender risk and without MCV is given by

$$
\begin{aligned}
V_{0}= & \mathbf{E}^{\mathbb{Q}}\left[\mathrm{e}^{-\int_{0}^{N} r(s) d s}\left(1+\sum_{j=1}^{N} \max \left(F, \min \left(C, \alpha\left[R_{j}-1\right]\right)\right)\right)\right] \\
= & \mathbf{E}^{\mathbb{Q}}\left[\mathrm{e}^{-\int_{0}^{N} r(s) d s}\left(1+N F+\sum_{j=1}^{N}\left(\alpha\left(R_{j}-1\right)-F\right)^{+}-\sum_{j=1}^{N}\left(\alpha\left(R_{j}-1\right)-C\right)^{+}\right)\right] \\
= & \mathbf{E}^{\mathbb{Q}}\left[\mathrm{e}^{-\int_{0}^{N} r(s) d s}(1+N F)\right] \\
& +\sum_{j=1}^{N}\left\{\mathbf{E}^{\mathbb{Q}}\left[\mathrm{e}^{-\int_{0}^{N} r(s) d s}\left(\alpha\left(R_{j}-1\right)-F\right)^{+}\right]-\mathbf{E}^{\mathbb{Q}}\left[\mathrm{e}^{-\int_{0}^{N} r(s) d s}\left(\alpha\left(R_{j}-1\right)-C\right)^{+}\right]\right\}
\end{aligned}
$$

where $x^{+}=\max (0, x)$. The following theorem provides the asymptotic expansion formula for the price of simple Ratchet EIAs without early surrender and without MCV.

Theorem 1 The first order approximation of the price of N-year simple Ratchet EIAs without $M C V$ under the models (1) and (2) is given by

$$
V_{0}=L_{0}+\sigma L_{1}+o(\sigma)
$$

where

$$
\begin{aligned}
& L_{0}=Y_{0}+\sum_{j=1}^{N}\left\{\alpha C_{j} e^{\frac{\Sigma_{00}^{(j)}}{2}}\left[\Phi\left(G_{0}^{(j)}(C)\right)-\Phi\left(G_{0}^{(j)}(F)\right)\right]\right. \\
& \left.+Y_{0}(\alpha+F) \Phi\left(H_{0}^{(j)}(F)\right)-Y_{0}(\alpha+C) \Phi\left(H_{0}^{(j)}(C)\right)+C Y_{0}\right\} \\
& L_{1}=\sum_{j=0}^{N} \alpha\left\{C_{j} e^{\frac{\Sigma_{00}^{(j)}}{2}}\left[D^{1} \Phi\left(G_{0}^{(j)}(C)\right)\left(H_{0}^{(j)}(C)\right)-D^{1} \Phi\left(G_{0}^{(j)}(F)\right)\left(H_{0}^{(j)}(F)\right)\right]\right. \\
& +C_{j}\left(a_{3}^{(j)}+a_{4}^{(j)}\right) e^{\frac{\Sigma_{00}^{(j)}}{2}}\left[\sqrt{\frac{\Sigma_{00}^{(j)}}{2 \pi}}\left(e^{-\frac{\left(G_{0}^{(j)}(C)\right)^{2}}{2}}-e^{-\frac{\left(G_{0}^{(j)}(F)\right)^{2}}{2}}\right)\right. \\
& \left.\left.+\Sigma_{00}^{(j)}\left[\Phi\left(G_{0}^{(j)}(F)\right)-\Phi\left(G_{0}^{(j)}(C)\right)\right]\right]\right\} \\
& +\sum_{j=0}^{N}(F+\alpha) Y_{0}\left[D^{1} \Phi\left(H_{0}^{(j)}(F)\right)\left(H_{12}^{(j)}(F)\right)+a_{5}^{(j)} \sqrt{\frac{\Sigma_{00}^{(j)}}{2 \pi}} e^{-\frac{\left(H_{0}^{(j)}(F)\right)^{2}}{2}}\right] \\
& -\sum_{j=0}^{N}(C+\alpha) Y_{0}\left[D^{1} \Phi\left(H_{0}^{(j)}(C)\right)\left(H_{12}^{(j)}(C)\right)+a_{5}^{(j)} \sqrt{\frac{\Sigma_{00}^{(j)}}{2 \pi}} e^{-\frac{\left(H_{0}^{(j)}(C)\right)^{2}}{2}}\right]
\end{aligned}
$$


with

$$
\begin{aligned}
& Y_{0}=\exp \left(-\int_{0}^{N} r^{(0)}(t) d t\right), r^{(0)}(t)=e^{-k t}[r(0)-\theta-\varphi(0)]+\theta+\varphi(t), \\
& C_{j}=\exp \left[-\int_{0}^{j-1} r^{(0)}(s) d s-\int_{j}^{N} r^{(0)}(s) d s-\frac{1}{2} \sigma_{S}^{2}\right], \\
& \Theta_{0}^{(j)}(x)=\log \left(\frac{x+\alpha}{\alpha}\right)-\int_{j-1}^{j}\left[r^{(0)}(s)-\frac{\sigma_{S}^{2}}{2}\right] d s, \quad \Theta_{12}^{(j)}(x)=-\left[\frac{\Sigma_{01}^{(j)}}{\Sigma_{00}^{(j)}}\right] \Theta_{0}^{(j)}(x), \\
& G_{0}^{(j)}(x)=\frac{\Theta_{0}^{(j)}(x)-\Sigma_{00}^{(j)}}{\sqrt{\Sigma_{00}^{(j)}}}, \quad H_{0}^{(j)}(x)=\frac{\Theta_{0}^{(j)}(x)}{\sqrt{\Sigma_{00}^{(j)}}}, \quad H_{12}^{(j)}(x)=\frac{\Theta_{12}^{(j)}(x)}{\sqrt{\Sigma_{00}^{(j)}}}, \quad a_{i}^{(j)}=\frac{\Sigma_{0 i}^{(j)}}{\Sigma_{00}^{(j)}},
\end{aligned}
$$

and $D^{n} \Phi(x)$ is the $n$th derivative of the standard Normal distribution function, $\Sigma_{n m}^{(j)}, n, m=$ $0, \cdots, 5$ is the covariance of $N_{j n}$ and $N_{j m}$, and

$$
\begin{aligned}
N_{j 0}= & \int_{j-1}^{j} \sigma_{S} d B_{1}(s)=\sigma_{S}\left[B_{1}(j)-B_{1}(j-1)\right], \\
N_{j 1}= & \int_{j-1}^{j} A_{1}(s) d s, \quad N_{j 3}=\int_{0}^{j-1} \int_{s}^{j-1} e^{-k(t-s)} \sqrt{r^{(0)}(s)-\varphi(s)} d t d B_{2}(s), \\
N_{j 4}= & \int_{j}^{N} A_{1}(s) d s=\int_{0}^{j} \int_{j}^{N} e^{-k(t-s)} \sqrt{r^{(0)}(s)-\varphi(s)} d t d B_{2}(s) \\
& +\int_{j}^{N} \int_{s}^{N} e^{-k(t-s)} \sqrt{r^{(0)}(s)-\varphi(s)} d t d B_{2}(s), \\
N_{j 5}= & \int_{0}^{N} A_{1}(s) d s=\int_{0}^{N} \int_{s}^{N} e^{-k(t-s)} \sqrt{r^{(0)}(s)-\varphi(s)} d t d B_{2}(s) .
\end{aligned}
$$

The proof of this theorem will be given in the Appendix.

\section{A lattice method for early surrender risk and MCV}

\subsection{The binomial tree for $\mathrm{S}$ and $\mathrm{r}$}

This section is devoted to describe the construction of a bivariate lattice structure proposed by Wei [18] following the Nelson and Ramaswamy [16] technique. This procedure allows us to build a binomial computational simple tree that approximates the evolution of the processes $S$ and $r$ in the geometric Brownian motion with $\mathrm{CIR}++$ stochastic interest rates model described by equations (1), (2). We use for the interest rate $r$ the two-steps procedure introduced in [10].

The first step consists in the construction of a binomial tree for the process $x$. Following [16] we consider the transformation of $x: X=2 \sqrt{x} / \sigma$, in order to introduce a diffusion with unit variance. By Ito's Lemma

$$
d X(t)=\mu_{X} d t+d B_{2}(t), \quad X(0)=2 \sqrt{x_{0}} / \sigma,
$$

where $\mu_{X}=\frac{k\left(4 \theta-X^{2}(t) \sigma^{2}\right)-\sigma^{2}}{2 X(t) \sigma^{2}}$. 
To construct the discrete approximations of the processes $X$ we divide the time to maturity $N$ into $n$ intervals of length $\Delta t=N / n$. Since $X$ is a process with unit variance we use a random walk approximation of the standard Brownian motion. As usual, a binomial tree may be considered to describe the evolution of the discrete approximating processes. We label $(0,0)$ the starting node where the $X$-process has value $X(0)$. After $i$ time steps $(0 \leq i \leq n) X$ may be located at one of the nodes $(i, k)(k=0, \ldots, i)$ corresponding to the values

$$
X_{i, k}=X_{0}+(2 k-i) \sqrt{\Delta t} .
$$

Transition probabilities have to be specified to assure the matching of the local drift and of the local variance between the discrete and the continuous model of $X$. This will guarantee that the discretized process converges in distribution to the corresponding diffusion. To do this we have to take into account that in some regions of the tree it may happen that multiple jumps are needed to satisfy properly the matching conditions. According to [16], we define $k_{d}$ as the largest integer $k^{*}$ such that $X_{i, k}+\mu_{X} \Delta t \geq X_{i+1, k^{*}}$ and $k_{u}:=k_{d}+1$. The transition probabilities are then given by

$$
p_{i, k}=\frac{\mu_{X} \Delta t+X_{i, k}-X_{i+1, k_{d}}}{X_{i+1, k_{u}}-X_{i+1, k_{d}}} .
$$

Remark 1 In order to avoid possible exits from the lattice structure we need also the following additional changes: when $k_{d}<0$ we set $k_{d}=0$ and $p_{i, k}=0$, when $k_{d}>i$ we set $k_{d}=i$ and $p_{i, k}=1$.

The second step consists in a shift of the tree which allows to perfectly reproduce the market zero-coupon bonds structure. We compute $r(t)$ using the shift $\varphi(i \Delta t)$ to get $r_{i, k}=x_{i, k}+\varphi(i \Delta t)$ for all the nodes of the tree. The displacement $\varphi$ is computed in order to be coherent with the market zero-coupon curve at time 0 (see [10] and [3]).

In a similar way, we consider the following transformation $Y(t)$ of $S(t)$

$$
Y(t)=\frac{\frac{\log S(t)}{\sigma_{S}}-\rho X(t)}{\sqrt{1-\rho^{2}}} .
$$

Standard calculations show that the process $Y(t)$ has unit variance. Furthermore $Y(t)$ and $X(t)$ have null covariance. We can construct a recombining tree $Y_{i, j}$ for $Y$ in the same way as in the case of the process $X$.

Finally, we consider a bivariate tree obtained by merging the two univariate binomial tree for the state variables $X$ and $Y$. At each time step $i$ we consider $(i+1)^{2}$ nodes that we label $(i, j, k)$ corresponding to the values $X_{i, k}$ and $Y_{i, j}(k, j=0, \ldots, i)$. Starting from the node $(i, j, k)$, in consideration of possible multiple jumps and taking into account the tree structure, the process may reach one of the four nodes:

$$
\left(i+1, j_{u}, k_{u}\right), \quad\left(i+1, j_{u}, k_{d}\right), \quad\left(i+1, j_{d}, k_{u}\right), \quad\left(i+1, j_{d}, k_{d}\right),
$$

with probabilities

$$
p_{u u}, \quad p_{u d}, \quad p_{d u}, \quad p_{d d},
$$

respectively. Here $j_{u}, j_{d}, k_{u}, k_{d}$ are the indexes related to the number of multiple jumps on the tree in the $Y$ and $X$ directions, and $p_{u u}, p_{u d}, p_{d u}, p_{d d}$ are the transition probabilities computed using the orthogonality of the two processes.

The tree for the joint evolution of the processes $r$ and $S$ is derived simply by applying a suitable transformation to the discrete scheme just defined. Indeed, to each node of the tree $(i, j, k)$ it corresponds the value

$$
r_{i, k}= \begin{cases}\frac{X_{i, k}^{2} \sigma_{r}^{2}}{4}+\varphi(i \Delta t) & \text { if } X_{i, k}>0 \\ \varphi(i \Delta t) & \text { otherwise }\end{cases}
$$


and the value

$$
S_{i, j, k}=\exp \left[\sigma_{S}\left(\sqrt{1-\rho^{2}} Y_{i, j}+\rho X_{i, k}\right)\right] .
$$

We refer to [18] and [4] for a more detailed description of this lattice structure.

Based on the results of [2], [6], [14], [16] it follows that the approximating bivariate model defined above converges weakly to the corresponding bivariate continuous diffusion.

\subsection{The lattice algorithm}

The use of a tree allows an easy treatment of the early surrender risk. In fact, with this lattice procedure we can evaluate, at each reset date, the continuation value, i.e. the expected value of the discounted cash flow obtained not surrendering the contract, and then we can compare it with the payoff of the early surrender case. We solve the problem of pricing simple Ratchet EIAs with early surrender risk using the bivariate tree introduced in the previous section and the framework of the singular points introduced in [7].

For our purpose we need to evaluate, at every node of the tree, the price function $v$ that provides, for all possible admissible value of the running sum $Z$, the price of the contract. Since $v$ will depends on the nodes of the tree we use the notation $v_{i, j, k}(Z)$, where $i$ is the time index, $j, k$ are the indexes related to the underlying asset and the interest rate. Using a backward procedure the price function $v$ can be easily computed at maturity, in fact if $Z$ is the running $\operatorname{sum} v_{n, j, k}(Z)$ is equal to $\phi\left(Z, G_{N}\right)$.

From the knowing of $v$ at maturity we have to construct backward all the values of $v$ along the tree. During the backward procedure, at each reset date we need to compare the expected value of the discounted cash flow obtained not surrendering the contract with the payoff of the early surrender case. Moreover we need to consider the condition related to the jump of the running sum at each reset date. Finally $v_{0,0,0}(1)$ will provide the price of the contract in the discrete setting.

In order to construct a feasible backward procedure, we use the singular points technique that is based on the remark that $v_{i, j, k}(Z)$ can be treated as piecewise linear continuous function at every nodes of the tree. Such piecewise linear function represents the linear extension of the discrete values achieved by $v_{i, j, k}(Z)$. A piecewise linear continuous function $f(x)(x \in \mathbb{R})$ is completely characterized by the coordinates of the points $\left(x_{i}, f\left(x_{i}\right)\right)$ where it changes the slope of the function, such points are called the "singular points" of $f$. To stress the dependence on $i, j, k$, the singular points of $v$ will be denoted by $\left(Z_{i, j, k}^{l}, P_{i, j, k}^{l}\right)$. Here the index $l$ (again depending on $i, j, k$ ) counts the number of singular points of $v_{i, j, k}(Z)$. So $v$ is the linear piecewise function which joints the singular points, hence $v_{i, j, k}\left(Z_{i, j, k}^{l}\right)=P_{i, j, k}^{l}$ for every $l$. The function $v_{i, j, k}(Z)$ can be constructed by linearity from these values. The main idea of the procedure is that the calculation of the singular points at every node of the tree at time $i$ can be obtained directly from the knowing of all the singular points at time $i+1$, allowing the construction of a backward procedure. Moreover, although the number of singular points increases very fast during the backward procedure, we can reduce them by controlling the error involved in the elimination procedure. This reduction, joined with an important symmetry remark that reduces drastically the computations (see Remark 2), will be the more important elements needed for obtaining a feasible procedure. Now we describe it in details.

We assume that the difference between two observation times is constant and equal to one year and we denote by $m$ the number of steps of the binomial tree in every annual period. Therefore the total number of steps of the binomial tree is $n=m N$. The tree method that we propose consists in evaluating at every time step $i=0,1, . ., n$ the price of the simple Ratchet EIAs contract for every possible choice of the running sum $Z$ defined in (3).

We proceed now to the description of the simple Ratchet price value function $v_{i, j, k}(Z), i=$ $0, \ldots, n$ and $j, k=0, \ldots, i$, in terms of the running sum $Z$, at every node $(i, j, k)$ of the tree structure. 
The simple Ratchet price value $v$ at maturity, as function of the running $\operatorname{sum} Z$, is continuously defined by

$$
v_{n, j, k}(Z)=\phi\left(Z, G_{N}\right)
$$

where $\phi$ is the payoff function (4). $v_{n, j, k}$ is a piecewise linear function, defined in $[1+N F, 1+N C]$, characterized by the three singular points $\left(Z_{n, j, k}^{l}, P_{n, j, k}^{l}\right), l=1,2,3\left(Z_{n, j, k}^{l}\right.$ is the running sum, $P_{n, j, k}^{l}$ is the corresponding simple Ratchet price) given by: $\left(1+N F, G_{N}\right),\left(G_{N}, G_{N}\right),(1+N C, 1+$ $N C)$. Remark that when $G_{N} \notin[1+N F, 1+N C], v_{n, j, k}$ is constant so we have just two singular points.

Consider now the time step $n-m$ corresponding to the reset date $N-1$. Given a running sum $Z$ the corresponding simple Ratchet price depends on the returns of the underlying asset during the time period $[N-1, N]$. We obtain the price at each node at time $n-m$ using a backward induction procedure of $\mathrm{m}$ steps starting from an appropriate terminal condition.

In order to describe the procedure let us fix a node $(n-m, \tilde{j}, \tilde{k})$ at the time step $n-m$. We consider a new tree (called local tree), starting from the node $(n-m, \tilde{j}, \tilde{k})$, whose nodes are $(n-m+i, \tilde{j}+j, \tilde{k}+k)$, where $i=0, \ldots, m ; j, k=0, \ldots, i$. From the generic node $(n-m+i, \tilde{j}+j, \tilde{k}+k)$ of the local tree the process can reach the nodes

$\left(n-m+i+1, j_{u}, k_{u}\right), \quad\left(n-m+i+1, j_{u}, k_{d}\right), \quad\left(n-m+i+1, j_{d}, k_{u}\right), \quad\left(n-m+i+1, j_{d}, k_{d}\right)$

with probabilities $p_{u u}, p_{u d}, p_{d u}, p_{d d}$, respectively. The indexes $j_{u}, j_{d}, k_{u}, k_{d}$ and the probabilities $p_{u u}, p_{u d}, p_{d u}, p_{d d}$ are those determined in the previous section for the global tree (see (12)) except for one change: now the reached nodes cannot exit from the local tree. Hence multiple jumps have to be limited to the local tree, therefore if $k_{u}>\tilde{k}+i$ then we have to change such a value and the corresponding probabilities setting $k_{u}:=\tilde{k}+i$ and $p_{n-m+i, \tilde{k}+k}=1$, while if $k_{d}<\tilde{k}$ we set $k_{d}=\tilde{k}$ and $p_{n-m+i, \tilde{k}+k}=0$. A corresponding adjustment is needed for $j_{u}, j_{d}$ as well.

Given a terminal node $(n, \tilde{j}+j, \tilde{k}+k)$ of the local tree starting from the node $(n-m, \tilde{j}, \tilde{k})$, the Ratchet interest rate here evaluated is given by

$$
\hat{R}=\min \left(C, \max \left(F, \alpha\left[\frac{S_{(n, \tilde{j}+j, \tilde{k}+k)}}{S_{(n-m, \tilde{j}, \tilde{k})}}-1\right]\right)\right) .
$$

Let $v_{n, \tilde{j}+j, \tilde{k}+k}^{-}$the simple Ratchet value function at the terminal node $(n, \tilde{j}+j, \tilde{k}+k)$ one instant before the reset date $N$, i.e. before to consider the Ratchet interest rate of the last year. Following Wilmott [19], no-arbitrage considerations lead to the following jump condition at each node $(n, \tilde{j}+$ $j, \tilde{k}+k)$ reachable with $m$ steps and starting from the node $(n-m, \tilde{j}, \tilde{k})$ :

$$
v_{n, \tilde{j}+j, \tilde{k}+k}^{-}(Z)=v_{n, \tilde{j}+j, \tilde{k}+k}(Z-\hat{R}) .
$$

$v_{n, \tilde{j}+j, \tilde{k}+k}^{-}$is now defined in the domain $[1+(N-1) F, 1+(N-1) C]$.

In summary, we can compute the price of simple Ratchet contract at each node $(n-m, \tilde{j}, \tilde{k})$, using the following procedure:

- At time $n$ (time $m$ of the local tree) we initialize the local tree at all the nodes $(n, \tilde{j}+j, \tilde{k}+k)$ taking the singular points of the global tree at these nodes and shifting the abscissa of the return $\hat{R}$. The singular points become

$$
\left(Z_{n, \tilde{j}+j, \tilde{k}+k}^{1}-\hat{R}, P_{n, \tilde{j}+j, \tilde{k}+k}^{1}\right),\left(Z_{n, \tilde{j}+j, \tilde{k}+k}^{2}-\hat{R}, P_{n, \tilde{j}+j, \tilde{k}+k}^{2}\right),\left(Z_{n, \tilde{j}+j, \tilde{k}+k}^{3}-\hat{R}, P_{n, \tilde{j}+j, \tilde{k}+k}^{3}\right) .
$$


- At time $n-1$ (time $m-1$ of the local tree) we have to evaluate the value function $v$ at the nodes $(n-1, \tilde{j}+j, \tilde{k}+k)$ for all $j, k=0, \ldots, m-1$. To this end we have to consider the set of the abscissae of all the singular points of the price functions $v_{n, j_{u}, k_{u}}^{-}(Z), v_{n, j_{u}, k_{d}}^{-}(Z)$, $v_{n, j_{d}, k_{u}}^{-}(Z), v_{n, j_{d}, k_{d}}^{-}(Z)$. Such set becomes the set of the abscissae of the singular points of $v_{n-1, \tilde{j}+j, \tilde{k}+k}(Z)$. The associated prices can be computed by

$v_{n-1, \tilde{j}+j, \tilde{k}+k}(Z)=e^{-r_{i, k} \Delta t}\left[p_{u u} v_{n, j_{u}, k_{u}}^{-}(Z)+p_{u d} v_{n, j_{u}, k_{d}}^{-}(Z)+p_{d u} v_{n, j_{d}, k_{u}}^{-}(Z)+p_{d d} v_{n, j_{d}, k_{d}}^{-}(Z)\right]$.

$v_{n, j_{u}, k_{u}}^{-}(Z)$ can be obtained by linear interpolation since $v_{n, \tilde{j}+j, \tilde{k}+k}^{-}$is a piecewise linear continuous function. All the other values of the previous recursion formula are computed in the same way.

- At time $n-2, . ., n-m$ we use backwardly the same procedure of the previous step obtaining at the end the value function $v_{n-m, \tilde{j}, \tilde{k}}(Z)$ defined again in $(1+(N-1) F, 1+(N-1) C)$.

Remark 2 The previous evaluation has to be repeated for every $\tilde{j}, \tilde{k}=0, \ldots, n-m$. However, a simple trick permits us to reduce in a meaningful way the computations. At first let us remark that $\hat{R}$ (see (13)) depends on $j, k$ but not on the indexes $\tilde{j}, \tilde{k}$. Moreover the probabilities $p_{u u}, p_{u d}, p_{d u}, p_{d d}$ evaluated on the local tree are independent on $\tilde{j}$. Observe that, on the contrary, in the global tree the probabilities are dependent on $\tilde{j}$ because of the presence of possible multiple jumps and of the corresponding treatment described in Remark 1 . We can conclude that the function $v_{n-m, j, \tilde{k}}(Z)$ is the same for all the choices $j=0, \ldots, n-m$ so that the use of the local tree allows us to reduce the complexity of the procedure of one order.

After the computation of $v_{n-m, j, k}(Z)$ for all $j, k=0, \ldots, n-m$, we then proceed iteratively at each node $(i, j, k), i=n-2 m, n-3 m, . ., m, 0$, of the global tree. At each reset date, we use again: a local tree, the appropriate terminal no-arbitrage condition (14) at time $i+m$, the backward induction procedure and the complexity reduction presented in Remark 2 . At the end of the procedure we obtain $v_{0,0,0}(1)$ which is the discrete lattice value of the simple Ratchet EIAs with $n$ steps and without early surrender risk.

The knowing of the price function at every node of our tree, allows us to treat in an easy way the case when the early surrender risk is embedded into the simple Ratchet EIAs contract. In fact we have to check at time $i=n-m, n-2 m, . ., m$, corresponding at each reset date $N-1, \ldots, 1$, the convenience of the early surrender option. Hence, the only difference with respect to the no early surrender case is that at the reset date, before shifting the abscissa of the singular points we have to modify the price function $v_{i, j, k}(Z)$ taking

$$
\max \left(\phi\left(Z, G_{i}\right), v_{i, j, k}(Z)\right)
$$

where $\phi$ is the payoff function (6). Such new functions, denoted for sake of simplicity, again by $v_{i, j, k}(Z)$ are still piecewise linear and can be easily computed. With these changes $v_{0,0,0}(1)$ provides the lattice value of the simple Ratchet EIAs with early surrender risk, associated to the tree with $n$ steps.

The technique previously presented is convergent to the value of the Ratchet contract obtained by the continuous bivariate model (1), (2). However, it can be problematic from a computational point of view because of the high number of singular points generated by the procedure. We can use the argument proposed in [7], based on the the convexity of the piecewise linear functions $v_{i, j, k}$, to reduce drastically such number controlling the error involved in the elimination procedure. Let us remark that deleting some internal points from the sequence of the singular points which characterizes $v_{i, j, k}$, the new function is still piecewise linear and convex. If we fix a given level 
$h>0$ for the error, we can easily eliminate singular points in order to obtain a new piecewise linear function which differs from the original one at most for $h$. Repeating this process at all the nodes of the tree the difference between $v_{0,0,0}(1)$ and the new approximating value is less than $n h$. So it is sufficient to choose the maximal level of error $h$ depending on $n$ and such that $h(n) n \rightarrow 0$ as $n \rightarrow \infty$, for obtaining the convergence of the values obtained by the elimination procedure to the continuous value. For a detailed discussion we refer to [7] (see the argument for obtaining the upper estimates) and [4].

\section{$6 \quad$ Numerical results}

In this section we will test the algorithms presented in Section 4 and Section 5 for computing the price of the simple Ratchet EIAs under CIR ++ stochastic interest rates.

We choose the parameters of the contract in a similar way as in [11]: the MCV parameters $\beta=0,0.9,1, g=0.03$, the partecipation rate $\alpha=0.9$, the local floor $F=0$, the local cap $C=0.16,0.20$. The maturity $N$ is 7 years.

We assume that the policyholder receives, in the early surrender case, the amount

$$
\phi\left(Z_{i}, G_{i}\right)=\max \left(\xi\left(i, N_{c}\right) Z_{i}, G_{i}\right), \quad i=1, \ldots, N .
$$

According to 2009 Annuity Fact Book ([20]) we take $N_{c}=5$ and we assume that the charge of the cancelation decreases every years of $1 \%$, vanishing at the year $N_{c}+1$. Therefore

$$
\xi\left(i, N_{c}\right)=1-\max \left\{\frac{N_{c}+1-i}{100}, 0\right\}, \quad i=1, \ldots, N .
$$

The parameters of the continuous bivariate model defined in equations (1), (2) are: $S_{0}=100$, $\sigma_{S}=0.2, x_{0}=0.04, \theta=0.05, k=0.5, \sigma=0.08,0.16, \rho=-0.3,0,0.3$. According to [11] we consider the following initial instantaneous forward curve $f(0, t)=0.04+0.0045 t+0.00015 t^{2}$ and the corresponding shift

$$
\varphi(t)=f(0, t)-\frac{2 k \theta\left(e^{t h}-1\right)}{2 h+(k+h)\left(e^{t h}-1\right)}-x_{0} \frac{4 h^{2} e^{t h}}{\left[2 h+(k+h)\left(e^{t h}-1\right)\right]^{2}}
$$

with $h=\sqrt{k^{2}+2 \sigma^{2}}$ (see [3]).

We apply the expansion method of Section 4 and the singular points algorithm presented in Section 5 for computing an estimate of the simple Ratchet EIAs price value. In the tables we will consider $n=140,280,420$ time steps.

The benchmark values are obtained by using a Monte Carlo method with a large number of simulations (1 million) using the Alfonsi [1] discretization scheme for the CIR process with 700 discretization time steps. This method provide a Monte Carlo weak second-order scheme for the Cox-Ingersoll-Ross process.

In Tables 1 we report different simple Ratchet prices without early surrender risk and without $\operatorname{MCV}(\beta=0)$. In Table 2 we report prices of simple Ratchet EIAs with MCV $(\beta=0.9,1)$ without surrender options. In Table 3 we report prices of simple Ratchet EIAs with MCV and with early surrender risk using the proposed tree method. No benchmarks are available in the early surrender case.

Table 1 Price EIA without MCV $(\beta=0)$ and without early surrender risk 


\begin{tabular}{cccccc}
$\sigma$ & Cap & Method & $\rho=-0.3$ & $\rho=0$ & $\rho=0.3$ \\
\hline 0.08 & $16 \%$ & Exp & 0.9985 & 0.9971 & 0.9957 \\
& & Tree $n=140$ & 1.0015 & 0.9956 & 0.9986 \\
& & Tree $n=280$ & 1.0009 & 0.9956 & 0.9980 \\
& & Tree $n=420$ & 1.0007 & 0.9978 & 0.9978 \\
& & MC & 0.9998 & 0.9984 & 0.9970 \\
\hline 0.08 & $20 \%$ & Exp & 1.0421 & 1.0412 & 1.0403 \\
& & Tree $n=140$ & 1.0462 & 1.0394 & 1.0433 \\
& & Tree $n=280$ & 1.0452 & 1.0417 & 1.0424 \\
& & Tree $n=420$ & 1.0448 & 1.0423 & 1.0420 \\
& & MC & 1.0438 & 1.0424 & 1.0410 \\
\hline 0.16 & $16 \%$ & Exp & 0.9946 & 0.9917 & 0.9890 \\
& & Tree $n=140$ & 1.0017 & 0.9946 & 0.9961 \\
& & Tree $n=280$ & 1.0011 & 0.9946 & 0.9956 \\
& & Tree $n=420$ & 1.0009 & 0.9968 & 0.9954 \\
& & MC & 1.0003 & 0.9976 & 0.9948 \\
\hline 0.16 & $20 \%$ & Exp & 1.0377 & 1.0358 & 1.0034 \\
& & Tree $n=140$ & 1.0464 & 1.0384 & 1.0408 \\
& & Tree $n=280$ & 1.0454 & 1.0407 & 1.0399 \\
& & Tree $n=420$ & 1.0450 & 1.0414 & 1.0395 \\
& & MC & 1.0442 & 1.0417 & 1.0388 \\
\hline & & & & &
\end{tabular}


Table 2 Price EIA with MCV and without early surrender risk

\begin{tabular}{ccccccc}
$\sigma$ & Cap & $\beta$ & Method & $\rho=-0.3$ & $\rho=0$ & $\rho=0.3$ \\
\hline 0.16 & $16 \%$ & 0.9 & Tree $n=140$ & 1.0025 & 0.9959 & 0.9974 \\
& & & Tree $n=280$ & 1.0019 & 0.9957 & 0.9969 \\
& & & Tree $n=420$ & 1.0017 & 0.9979 & 0.9967 \\
& & & MC & 1.0010 & 0.9986 & 0.9960 \\
\hline 0.16 & $16 \%$ & 1 & Tree $n=140$ & 1.0075 & 1.0022 & 1.0040 \\
& & & Tree $n=280$ & 1.0072 & 1.0018 & 1.0037 \\
& & & Tree $n=420$ & 1.0072 & 1.0020 & 1.0037 \\
& & & MC & 1.0059 & 1.0043 & 1.0024 \\
\hline 0.16 & $20 \%$ & 0.9 & Tree $n=140$ & 1.0472 & 1.0397 & 1.0421 \\
& & & Tree $n=280$ & 1.0462 & 1.0418 & 1.0412 \\
& & & Tree $n=420$ & 1.0458 & 1.0424 & 1.0408 \\
& & & MC & 1.0449 & 1.0426 & 1.0400 \\
\hline 0.16 & $20 \%$ & 1 & Tree $n=140$ & 1.0514 & 1.0451 & 1.0476 \\
& & & Tree $n=280$ & 1.0506 & 1.0469 & 1.0469 \\
& & & Tree $n=420$ & 1.0504 & 1.0475 & 1.0467 \\
& & & MC & 1.0490 & 1.0474 & 1.0454 \\
\hline
\end{tabular}

Table 3 Price EIA with MCV and with early surrender risk

\begin{tabular}{ccccccc}
\hline$\sigma$ & Cap & $\beta$ & Method & $\rho=-0.3$ & $\rho=0$ & $\rho=0.3$ \\
0.16 & $16 \%$ & 0.9 & Tree $n=140$ & 1.0403 & 1.0379 & 1.0415 \\
& & & Tree $n=280$ & 1.0399 & 1.0376 & 1.0412 \\
& & & Tree $n=420$ & 1.0398 & 1.0394 & 1.0411 \\
\hline 0.16 & $16 \%$ & 1 & Tree $n=140$ & 1.0558 & 1.0534 & 1.0535 \\
& & & Tree $n=280$ & 1.0554 & 1.0523 & 1.0531 \\
& & & Tree $n=420$ & 1.0554 & 1.0537 & 1.0532 \\
\hline 0.16 & $20 \%$ & 0.9 & Tree $n=140$ & 1.0785 & 1.0751 & 1.0802 \\
& & & Tree $n=280$ & 1.0776 & 1.0768 & 1.0794 \\
& & & Tree $n=420$ & 1.0773 & 1.0774 & 1.0791 \\
\hline 0.16 & $20 \%$ & \multirow{2}{*}{1} & Tree $n=140$ & 1.0879 & 1.0848 & 1.0870 \\
& & & Tree $n=280$ & 1.0871 & 1.0855 & 1.0864 \\
& & & Tree $n=420$ & 1.0868 & 1.0859 & 1.0861 \\
\hline
\end{tabular}

In Tables 4-6 we report some results on the break-even participation rate (BPR), which is defined to be the participation rate $\alpha$ at which the price of an EIA equals its notional principal $(1 \$)$. 
Table 4 BPRs EIA without MCV $(\beta=0)$ and without early surrender risk

\begin{tabular}{cccccc}
\hline$\sigma$ & Cap & Method & $\rho=-0.3$ & $\rho=0$ & $\rho=0.3$ \\
\hline 0.08 & $16 \%$ & Exp & 0.9119 & 0.9235 & 0.9360 \\
& & Tree $n=140$ & 0.8871 & 0.9579 & 0.9129 \\
& & Tree $n=280$ & 0.8933 & 0.9398 & 0.9163 \\
& & Tree $n=420$ & 0.8950 & 0.9151 & 0.9185 \\
& & MC & 0.9014 & 0.9127 & 0.9250 \\
\hline 0.08 & $20 \%$ & Exp & 0.7059 & 0.7058 & 0.7057 \\
& & Tree $n=140$ & 0.6881 & 0.7091 & 0.6958 \\
& & Tree $n=280$ & 0.6926 & 0.6977 & 0.6999 \\
& & Tree $n=420$ & 0.6937 & 0.7011 & 0.7009 \\
& & MC & 0.6974 & 0.7008 & 0.7046 \\
\hline 0.16 & \multirow{2}{*}{$16 \%$} & Exp & 0.9437 & 0.9711 & 1.0030 \\
& & Tree $n=140$ & 0.8856 & 0.9712 & 0.9354 \\
& & Tree $n=280$ & 0.8920 & 0.9489 & 0.9382 \\
& & Tree $n=420$ & 0.8936 & 0.9221 & 0.9411 \\
& & MC & 0.8980 & 0.9195 & 0.9447 \\
\hline 0.16 & $20 \%$ & Exp & 0.7260 & 0.7270 & 0.7280 \\
& & Tree $n=140$ & 0.6878 & 0.7124 & 0.7032 \\
& & Tree $n=280$ & 0.6923 & 0.7010 & 0.7071 \\
& & Tree $n=420$ & 0.6934 & 0.7041 & 0.7079 \\
& & MC & 0.6964 & 0.7027 & 0.7109 \\
\hline
\end{tabular}

Table 5 BPRs EIA with MCV and without early surrender risk

\begin{tabular}{ccccccc}
$\sigma$ & Cap & $\beta$ & Method & $\rho=-0.3$ & $\rho=0$ & $\rho=0.3$ \\
\hline 0.16 & $16 \%$ & 0.9 & Tree $n=140$ & 0.8788 & 0.9559 & 0.9246 \\
& & & Tree $n=280$ & 0.8854 & 0.9394 & 0.9266 \\
& & & Tree $n=420$ & 0.8865 & 0.9149 & 0.9297 \\
& & & MC & 0.8921 & 0.9117 & 0.9347 \\
\hline 0.16 & $16 \%$ & 1 & Tree $n=140$ & 0.8388 & 0.8691 & 0.8625 \\
& & & Tree $n=280$ & 0.8425 & 0.8824 & 0.8689 \\
& & & Tree $n=420$ & 0.8430 & 0.8706 & 0.8686 \\
& & & MC & 0.8523 & 0.8639 & 0.8786 \\
\hline 0.16 & $20 \%$ & 0.9 & Tree $n=140$ & 0.6836 & 0.7045 & 0.6964 \\
& & & Tree $n=280$ & 0.6875 & 0.6941 & 0.7002 \\
& & & Tree $n=420$ & 0.6886 & 0.6979 & 0.7009 \\
& & & MC & 0.6925 & 0.6978 & 0.7048 \\
\hline 0.16 & $20 \%$ & 1 & Tree $n=140$ & 0.6589 & 0.6640 & 0.6636 \\
& & & Tree $n=280$ & 0.6609 & 0.6680 & 0.6652 \\
& & & Tree $n=420$ & 0.6614 & 0.6666 & 0.6655 \\
& & & MC & 0.6676 & 0.6689 & 0.6719 \\
\hline
\end{tabular}

Table 6 BPRs EIA with MCV and with early surrender risk 


\begin{tabular}{ccccccc}
$\sigma$ & Cap & $\beta$ & Method & $\rho=-0.3$ & $\rho=0$ & $\rho=0.3$ \\
\hline 0.16 & $16 \%$ & 0.9 & Tree $n=140$ & 0.5952 & 0.6037 & 0.5769 \\
& & & Tree $n=280$ & 0.5975 & 0.5951 & 0.5784 \\
& & & Tree $n=420$ & 0.5988 & 0.5951 & 0.5789 \\
\hline 0.16 & $16 \%$ & 1 & Tree $n=140$ & 0.3333 & 0.3372 & 0.3404 \\
& & & Tree $n=280$ & 0.3354 & 0.3385 & 0.3430 \\
& & & Tree $n=420$ & 0.3356 & 0.3401 & 0.3431 \\
\hline 0.16 & \multirow{2}{*}{$0 \%$} & 0.9 & Tree $n=140$ & 0.5252 & 0.5248 & 0.5076 \\
& & & Tree $n=280$ & 0.5272 & 0.5212 & 0.5093 \\
& & & Tree $n=420$ & 0.5281 & 0.5218 & 0.5096 \\
\hline 0.16 & $20 \%$ & 1 & Tree $n=140$ & 0.3216 & 0.3211 & 0.3246 \\
& & & Tree $n=280$ & 0.3229 & 0.3242 & 0.3263 \\
& & & Tree $n=420$ & 0.3232 & 0.3252 & 0.3262 \\
\hline
\end{tabular}

The previous data have been performed in double precision on a PC with processor Centrino 2 at $2.4 \mathrm{Ghz}$ with $4 \mathrm{~Gb}$ of RAM. The time of computation of the pricing algorithm in a selected case are reported in next table:

Table 7 Computation times for pricing EIA without early surrender risk.

\begin{tabular}{ccc}
\multicolumn{3}{c}{ Cap $=16 \%, \sigma=16 \%, \rho=0.3$} \\
\hline$\beta=0$ & Method & Time (in seconds) \\
& Exp & 0.22 \\
& Tree $n=140$ & 0.78 \\
& Tree $n=280$ & 11.3 \\
& Tree $n=420$ & 60.1 \\
\hline$\beta=0.9$ & Method & \\
& Tree $n=140$ & 2.43 \\
& Tree $n=280$ & 34.1 \\
& Tree $n=420$ & 171.6
\end{tabular}

\section{Conclusions}

We have introduced two different algorithms for pricing simple Ratchet EIAs in a geometric Brownian dynamics with the $\mathrm{CIR}++$ stochastic interest rates. The first method is based on expansion techniques and it allows to price simple Ratchet EIAs without early surrender risk and minimum contract value. The approximation is sharp for pricing purposes, but the behavior of the expansion is less precise for the computations of the BPRs. The second method is a tree algorithm treating general contract specifications like early surrender risk. Numerical comparisons indicate that the method is reliable and accurate.

\section{Acknowledgement}

We are indebted to Prof. Sheldon Lin for suggesting us the cancellation fee in the surrender case.

\section{Appendix. Proof of Theorem 1}

The proof is based on Theorem 4.5 of Kunitomo-Takahashi [13], which establishes a general result on an asymptotic expansion for pricing contingent claims in a general framework. The theorem applies to our case, so we just need to evaluate the coefficients $L_{0}, L_{1}$ of the expansion for pricing simple Ratchet EIAs without MCV and without early surrender risk.

This will be done in four steps. 
1. We derive the asymptotic expansion of interest rate by applying Theorem 4.4 in [13] which is based on the Watanabe-Yosida theory of Malliavin calculus.

2. We obtain the expression of the return of underlying asset using the results of step 1.

3. We derive an inequality, equivalent to $\left[R_{j} \geq \frac{x+\alpha}{\alpha}\right]$, necessary for the computation of the expectation $\mathbf{E}^{\mathbb{Q}}\left[\mathrm{e}^{-\int_{0}^{N} r(s) d s}\left(\alpha\left(R_{j}-1\right)-x\right)^{+}\right]$, by using the asymptotic expansion of Step 2 .

4. We obtain $L_{0}$ and $L_{1}$ by computing the expectations in $(7): \mathbf{E}^{\mathbb{Q}}\left[\mathrm{e}^{-\int_{0}^{N} r(s) d s}(1+N F)\right]$ and $\mathbf{E}^{\mathbb{Q}}\left[\mathrm{e}^{-\int_{0}^{N} r(s) d s}\left(\alpha\left(R_{j}-1\right)-x\right)^{+}\right]$for $j=1, \cdots, N$ and $x=F, C$.

\subsection{Step 1. Asymptotic expansion of the interest rate}

The CIR ++ interest rates model (2) can be rewritten in the integral form

$$
r(t)=r(0)+\varphi(t)-\varphi(0)+\int_{0}^{t} k(\theta-r(s)+\varphi(s)) d s+\sigma \int_{0}^{t} \sqrt{r(s)-\varphi(s)} d B_{2}(s) .
$$

From Theorem 4.4 in [13], $r(t)$ has an asymptotic expansion as

$$
r(t)=r^{(0)}(t)+\sum_{i=1}^{\infty} \sigma^{i} A_{i}(t)
$$

where $A_{i}(t)=\left.\frac{1}{i !} \frac{\partial^{i} r(t)}{\partial \sigma^{i}}\right|_{\sigma=0}, r^{(0)}(t)$ denotes $r(t)$ with volatility $\sigma=0$ and it satisfies the equation $r^{(0)}(t)=r(0)+\varphi(t)-\varphi(0)+\int_{0}^{t} k\left[\theta-r^{(0)}(s)+\varphi(s)\right] d s$, so that $r^{(0)}(t)=\mathrm{e}^{-k t}[r(0)-\theta-\varphi(0)]+\theta+\varphi(t)$. Differentiating (16) with respect to $\sigma$ leads to

$$
\frac{\partial r(t)}{\partial \sigma}=-\int_{0}^{t} k \frac{\partial r(s)}{\partial \sigma} d s+\int_{0}^{t} \sqrt{r(s)-\varphi(s)} d B_{2}(s)+\sigma \int_{0}^{t} \frac{1}{2 \sqrt{r(s)-\varphi(s)}} \frac{\partial r(s)}{\partial \sigma} d B_{2}(s),
$$

then letting $\sigma=0$ in the above equation, we obtain

$$
A_{1}(t)=-k \int_{0}^{t} A_{1}(s) d s+\int_{0}^{t} \sqrt{r^{(0)}(s)-\varphi(s)} d B_{2}(s)
$$

and solving the above equation we have

$$
A_{1}(t)=\int_{0}^{t} \mathrm{e}^{-k(t-s)} \sqrt{r^{(0)}(s)-\varphi(s)} d B_{2}(s)
$$

Note that $A_{1}(t)$ is a Gaussian process with $A_{1}(t) \sim N\left(0, \int_{0}^{t} \mathrm{e}^{-2 k(t-s)}\left[r^{(0)}(s)-\varphi(s)\right] d s\right)$.

\subsection{Step 2. Expansion expression of the equity value return}

By Itô's lemma, integrating (1) from 0 to $t$ one has

$$
\log [S(t) / S(0)]=\int_{0}^{t}\left[r(s)-\frac{1}{2} \sigma_{S}^{2}\right] d s+\int_{0}^{t} \sigma_{S} d B_{1}(s) .
$$

Substituting $r(s)$ with its expansion (17) we obtain

$$
S(t)=S(0) \exp \left\{\int_{0}^{t}\left[r^{(0)}(s)-\frac{1}{2} \sigma_{S}^{2}+\sum_{i=1}^{\infty} \sigma^{i} A_{i}(s)\right] d s+\int_{0}^{t} \sigma_{S} d B_{1}(s)\right\},
$$

$\mathrm{RR} \mathrm{n}^{\circ} 8034$ 
so that

$$
R_{j}=\frac{S(j)}{S(j-1)}=\exp \left\{\int_{j-1}^{j}\left[r^{(0)}(u)-\frac{1}{2} \sigma_{S}^{2}+\sum_{i=1}^{\infty} \sigma^{i} A_{i}(u)\right] d u+\sigma_{S}\left[B_{1}(j)-B_{1}(j-1)\right]\right\} .
$$

\subsection{Step 3. Equivalent inequality of $\left[R_{j} \geq \frac{x+\alpha}{\alpha}\right]$}

Since the payoff function of the simple Ratchet EIAs is not linear nor negative, its valuation should be done by taking into account the condition $R_{j} \geq \frac{x+\alpha}{\alpha}$ which is equivalent to

$$
\begin{aligned}
& \exp \left\{\int_{j-1}^{j}\left[r^{(0)}(u)-\frac{1}{2} \sigma_{S}^{2}+\sum_{i=1}^{\infty} \sigma^{i} A_{i}(u)\right] d u+\sigma_{S}\left[B_{1}(j)-B_{1}(j-1)\right]\right\} \geq \frac{x+\alpha}{\alpha} \\
\Leftrightarrow & \sigma_{S}\left[B_{1}(j)-B_{1}(j-1)\right] \geq \log \left(\frac{x+\alpha}{\alpha}\right)-\int_{j-1}^{j}\left[r^{(0)}(u)+\sum_{i=1}^{\infty} \sigma^{i} A_{i}(u)-\frac{\sigma_{S}^{2}}{2}\right] d u \\
\Leftrightarrow & \sigma_{S}\left[B_{1}(j)-B_{1}(j-1)\right] \geq \log \left(\frac{x+\alpha}{\alpha}\right)-\int_{j-1}^{j}\left[r^{(0)}(u)-\frac{\sigma_{S}^{2}}{2}\right] d u-\sum_{i=1}^{\infty} \sigma^{i} \int_{j-1}^{j} A_{i}(u) d u . \\
\Leftrightarrow & N_{j 0} \geq \log \left(\frac{x+\alpha}{\alpha}\right)-\int_{j-1}^{j}\left[r^{(0)}(u)-\frac{\sigma_{S}^{2}}{2}\right] d u-\sigma N_{j 1}-\sum_{i=2}^{\infty} \sigma^{i} \int_{j-1}^{j} A_{i}(u) d u
\end{aligned}
$$

where $N_{j 0}=\sigma_{S}\left[B_{1}(j)-B_{1}(j-1)\right], N_{j 1}=\int_{j-1}^{j} A_{1}(t) d t$. Obviously $N_{j 0}$ follows a Gaussian distribution, and by change of integration order and by (18) we have

$$
\begin{aligned}
N_{j 1} & =\int_{j-1}^{j} \int_{0}^{t} \mathrm{e}^{-k(t-s)} \sqrt{r^{(0)}(s)-\varphi(s)} d B_{2}(s) d t \\
& =\int_{0}^{j-1} \int_{j-1}^{j} \mathrm{e}^{-k(t-s)} \sqrt{r^{(0)}(s)-\varphi(s)} d t d B_{2}(s)+\int_{j-1}^{j} \int_{s}^{j} \mathrm{e}^{-k(t-s)} \sqrt{r^{(0)}(s)-\varphi(s)} d t d B_{2}(s) .
\end{aligned}
$$

Then the variances of $N_{j 0}$ and $N_{j 1}$ are $\Sigma_{00}^{(j)}=\operatorname{Var}\left[N_{j 0}\right]=\sigma_{S}^{2}$ and

$$
\Sigma_{11}^{(j)}=\operatorname{Var}\left[N_{j 1}\right]=\int_{0}^{j-1}\left(\int_{j-1}^{j} \mathrm{e}^{-k(t-s)} \sqrt{r^{(0)}(s)-\varphi(s)} d t\right)^{2} d s+\int_{j-1}^{j}\left(\int_{s}^{j} \mathrm{e}^{-k(t-s)} \sqrt{r^{(0)}(s)-\varphi(s)} d t\right)^{2} d s
$$

respectively. Their covariance is

$$
\Sigma_{01}^{(j)}=\operatorname{Cov}\left[N_{j 0}, N_{j 1}\right]=\sigma_{S} \rho \int_{j-1}^{j} \int_{s}^{j} \mathrm{e}^{-k(t-s)} \sqrt{r^{(0)}(s)-\varphi(s)} d t d s .
$$

By Gaussian distribution properties, we know that the conditional distribution

$$
\left.N_{j 1}\right|_{N_{j 0}=x} \sim N\left(\frac{\Sigma_{01}^{(j)}}{\Sigma_{00}^{(j)}} x, \Sigma_{11}^{(j)}-\frac{\Sigma_{01}^{(j)} \Sigma_{01}^{(j)}}{\Sigma_{00}^{(j)}}\right)
$$

hence $\left.N_{j 1}\right|_{N_{j 0}=x}=\frac{\Sigma_{01}^{(j)}}{\Sigma_{00}^{(j)}} x+N_{j 2} \sqrt{\left|\Sigma_{11}^{(j)}-\frac{\Sigma_{01}^{(j)} \Sigma_{01}^{(j)}}{\Sigma_{00}^{(j)}}\right|}$, where $N_{j 2} \sim N(0,1)$ is independent of $N_{j 0}$.

By replacing $N_{j 1}$ with $\frac{\Sigma_{01}^{(j)}}{\Sigma_{00}^{(j)}} N_{j 0}+N_{j 2} \sqrt{\left|\Sigma_{11}^{(j)}-\frac{\Sigma_{01}^{(j)} \Sigma_{01}^{(j)}}{\Sigma_{00}^{(j)}}\right|}$ in the equivalent inequality (21), we have that $R_{j} \geq \frac{x+\alpha}{\alpha}$ is equivalent to

$N_{j 0} \geq \log \left(\frac{x+\alpha}{\alpha}\right)-\int_{j-1}^{j}\left[r^{(0)}(s)-\frac{\sigma_{S}^{2}}{2}\right] d s-\sigma\left(\frac{\Sigma_{01}^{(j)}}{\Sigma_{00}^{(j)}} N_{j 0}+N_{j 2} \sqrt{\left|\Sigma_{11}^{(j)}-\frac{\Sigma_{01}^{(j)} \Sigma_{01}^{(j)}}{\Sigma_{00}^{(j)}}\right|}\right)-\sum_{i=2}^{\infty} \sigma^{i} \int_{j-1}^{j} A_{i}(s) d s$. 
Rearranging the above inequality we have

$N_{j 0}\left[1+\sigma \frac{\Sigma_{01}^{(j)}}{\Sigma_{00}^{(j)}}\right] \geq \log \left(\frac{x+\alpha}{\alpha}\right)-\int_{j-1}^{j}\left[r^{(0)}(s)-\frac{\sigma_{S}^{2}}{2}\right] d s-\sigma N_{j 2} \sqrt{\left|\Sigma_{11}^{(j)}-\frac{\Sigma_{01}^{(j)} \Sigma_{01}^{(j)}}{\Sigma_{00}^{(j)}}\right|}-\sum_{i=2}^{\infty} \sigma^{i} \int_{j-1}^{j} A_{i}(s) d s$

then by Taylor's expansion $\frac{1}{1+x}=\sum_{i=0}^{\infty}(-x)^{i}$, we have the equivalent inequality of $(21)$

$$
\begin{aligned}
& N_{j 0} \\
\geq & \frac{1}{1+\sigma \frac{\Sigma_{01}^{(j)}}{\Sigma_{00}^{(j)}}}\left\{\log \left(\frac{x+\alpha}{\alpha}\right)-\int_{j-1}^{j}\left[r^{(0)}(s)-\frac{\sigma_{S}^{2}}{2}\right] d s-\sigma N_{j 2} \sqrt{\left|\Sigma_{11}^{(j)}-\frac{\Sigma_{01}^{(j)} \Sigma_{01}^{(j)}}{\Sigma_{00}^{(j)}}\right|}-\sum_{i=2}^{\infty} \sigma^{i} \int_{j-1}^{j} A_{i}(s) d s\right\} \\
= & \sum_{k=0}^{\infty}\left(-\sigma \frac{\Sigma_{01}^{(j)}}{\Sigma_{00}^{(j)}}\right)^{k}\left\{\log \left(\frac{x+\alpha}{\alpha}\right)-\int_{j-1}^{j}\left[r^{(0)}(s)-\frac{\sigma_{S}^{2}}{2}\right] d s-\sigma N_{j 2} \sqrt{\left|\Sigma_{11}^{(j)}-\frac{\Sigma_{01}^{(j)} \Sigma_{01}^{(j)}}{\Sigma_{00}^{(j)}}\right|}-\sum_{i=2}^{\infty} \sigma^{i} \int_{j-1}^{j} A_{i}(s) d s\right\} \\
= & \Theta_{0}^{(j)}(x)+\sigma\left[N_{j 2} \Theta_{11}^{(j)}+\Theta_{12}^{(j)}(x)\right]+\sum_{k=2}^{\infty} \sigma^{k}\left[N_{j 2} \Theta_{k 1}^{(j)}+\Theta_{k 2}^{(j)}(x)\right]
\end{aligned}
$$

where

$\Theta_{0}^{(j)}(x)=\log \left(\frac{x+\alpha}{\alpha}\right)-\int_{j-1}^{j}\left[r^{(0)}(s)-\frac{\sigma_{S}^{2}}{2}\right] d s, \Theta_{11}^{(j)}=-\sqrt{\left|\Sigma_{11}^{(j)}-\frac{\Sigma_{01}^{(j)} \Sigma_{01}^{(j)}}{\Sigma_{00}^{(j)}}\right|}, \quad$ and $\quad \Theta_{12}^{(j)}(x)=-\frac{\Sigma_{01}^{(j)}}{\Sigma_{00}^{(j)}} \Theta_{0}^{(j)}(x)$

for $k=2,3, \cdots$

$\Theta_{k 1}^{(j)}=-\sqrt{\left|\Sigma_{11}^{(j)}-\frac{\Sigma_{01}^{(j)} \Sigma_{01}^{(j)}}{\Sigma_{00}^{(j)}}\right|}\left[-\frac{\Sigma_{01}^{(j)}}{\Sigma_{00}^{(j)}}\right]^{k-1}, \quad \Theta_{k 2}^{(j)}(x)=\left[-\frac{\Sigma_{01}^{(j)}}{\Sigma_{00}^{(j)}}\right]^{k} \Theta_{0}^{(j)}(x)-\sum_{i=1}^{k-1}\left[-\frac{\Sigma_{01}^{(j)}}{\Sigma_{00}^{(j)}}\right]^{k-1-i} \int_{j-1}^{j} A_{i+1}(s) d s$.

In the following calculation, we will ignore the higher order term in the right-hand side of the equivalent inequality (24) and the error term of the approximation converges to 0 when $\sigma \rightarrow 0$.

\subsection{Step 4. Computation of $L_{0}$ and $L_{1}$}

\subsubsection{Computation of $\mathbf{E}^{\mathbb{Q}}\left[\mathbf{e}^{-\int_{0}^{N} r(s) d s}\right]$}

By the asymptotic expression (17) for the interest rate $r(s)$ and by applying Taylor's expansion to function $\mathrm{e}^{-x}$ around $x=-\int_{0}^{N} r^{(0)}(s) d s$, we have

$$
\begin{aligned}
& \mathbf{E}^{\mathbb{Q}}\left[\mathrm{e}^{-\int_{0}^{N} r(s) d s}\right]=\mathbf{E}^{\mathbb{Q}}\left\{\mathrm{e}^{-\int_{0}^{N}\left[r^{(0)}(s)+\sum_{i=1}^{\infty} \sigma^{i} A_{i}(s)\right] d s}\right\} \\
= & \mathbf{E}^{\mathbb{Q}}\left\{\mathrm{e}^{-\int_{0}^{N} r^{(0)}(s) d s}\left[\sum_{k=0}^{\infty} \frac{1}{k !}\left(-\sum_{i=1}^{\infty} \sigma^{i} \int_{0}^{N} A_{i}(s) d s\right)^{k}\right]\right\}=\sum_{k=0}^{\infty} \sigma^{k} \mathbf{E}^{\mathbb{Q}}\left(Y_{k}\right),
\end{aligned}
$$

where the first three terms of the series $\left\{Y_{k} ; k=0,1, \cdots\right\}$ are given explicitly as follows:

$$
Y_{0}=\exp \left[-\int_{0}^{N} r^{(0)}(s) d s\right], \quad Y_{1}=-Y_{0} \int_{0}^{N} A_{1}(s) d s, \quad Y_{2}=Y_{0}\left[\frac{1}{2}\left(\int_{0}^{N} A_{1}(s) d s\right)^{2}-\int_{0}^{N} A_{2}(s) d s\right]
$$

$\mathrm{RR} \mathrm{n}^{\circ} 8034$ 
8.4.2 Computation of $\mathbf{E}^{\mathbb{Q}}\left[\mathbf{e}^{-\int_{0}^{N} r(s) d s}\left(\alpha\left(R_{j}-1\right)-x\right)^{+}\right]$, for $j=1, \cdots, N$ and $x=F, C$

Let $\mathbf{1}_{(\omega)}$ be the indicator function of the event $\omega$, we have

$$
\begin{aligned}
& \mathbf{E}^{\mathbb{Q}}\left[\mathrm{e}^{-\int_{0}^{N} r(s) d s}\left(\alpha\left(R_{j}-1\right)-x\right)^{+}\right] \\
= & \alpha \mathbf{E}^{\mathbb{Q}}\left[\mathrm{e}^{-\int_{0}^{N} r(s) d s}\left(R_{j}-\frac{\alpha+x}{\alpha}\right) \mathbf{1}_{\left(R_{j} \geq \frac{\alpha+x}{\alpha}\right)}\right] \\
= & \alpha \mathbf{E}^{\mathbb{Q}}\left[\mathrm{e}^{-\int_{0}^{N} r(s) d s} R_{j} \mathbf{1}_{\left(R_{j} \geq \frac{\alpha+x}{\alpha}\right)}\right]-(\alpha+x) \mathbf{E}^{\mathbb{Q}}\left[\mathrm{e}^{-\int_{0}^{N} r(s) d s} \mathbf{1}_{\left(R_{j} \geq \frac{\alpha+x}{\alpha}\right)}\right] .
\end{aligned}
$$

Applying the asymptotic expansion of the interest rate $r(s)$ and the return $R_{j}$ in section 8.1 and 8.2 , the term $\mathrm{e}^{-\int_{0}^{N} r(s) d s} R_{j}$ inside the first expectation of the above formula can be rewritten as follows:

$$
\begin{aligned}
& \mathrm{e}^{-\int_{0}^{N} r(s) d s} R_{j} \\
= & \exp \left\{-\int_{(0, j-1) \cup(j, N)}\left[r^{(0)}(s)+\sum_{i=1}^{\infty} \sigma^{i} A_{i}(s)\right] d s-\int_{j-1}^{j} \frac{1}{2} \sigma_{S}^{2} d s+N_{j 0}\right\} \\
= & \exp \left[-\int_{(0, j-1) \cup(j, N)} r^{(0)}(s) d s-\int_{j-1}^{j} \frac{1}{2} \sigma_{S}^{2} d s+N_{j 0}\right] \exp \left[-\sum_{i=1}^{\infty} \sigma^{i} \int_{(0, j-1) \cup(j, N)} A_{i}(s) d s\right]
\end{aligned}
$$

Applying Taylor's expansion to the function $\mathrm{e}^{-x}$ around $x=0$, we have

$$
\begin{aligned}
& \mathrm{e}^{-\int_{0}^{N} r(s) d s} R_{j} \\
= & \mathrm{e}^{-\int_{(0, j-1) \cup(j, N)} r^{(0)}(s) d s-\frac{1}{2} \sigma_{S}^{2}+N_{j 0}} \sum_{k=0}^{\infty} \frac{\left(-\sum_{i=1}^{\infty} \sigma^{i} \int_{(0, j-1) \cup(j, N)} A_{i}(s) d s\right)^{k}}{k !}=\sum_{k=0}^{\infty} \sigma^{k} X_{j k} .
\end{aligned}
$$

The first three terms of the series $\left\{X_{j k} ; k=0,1, \cdots\right\}$ are given by:

$$
\begin{aligned}
& X_{j 0}=\mathrm{e}^{-\int_{(0, j-1) \cup(j, N)} r^{(0)}(s) d s-\frac{1}{2} \sigma_{S}^{2}+N_{j 0}}=C_{j} \mathrm{e}^{N_{j 0}}, \quad X_{j 1}=-X_{j 0} \int_{(0, j-1) \cup(j, N)} A_{1}(s) d s, \\
& X_{j 2}=X_{j 0}\left[-\int_{(0, j-1) \cup(j, N)} A_{2}(s) d s+\frac{1}{2}\left(\int_{(0, j-1) \cup(j, N)} A_{1}(s) d s\right)^{2}\right]
\end{aligned}
$$

where $C_{j}=\exp \left[-\int_{(0, j-1) \cup(j, N)} r^{(0)}(s) d s-\frac{1}{2} \sigma_{S}^{2}\right]$ is a deterministic term which can be computed explicitly from the given model parameters.

Following similar arguments used for (25) and applying (27) to (26), we have

$$
\begin{aligned}
& \mathbf{E}^{\mathbb{Q}}\left[\mathrm{e}^{-\int_{0}^{N} r(s) d s}\left(\alpha\left(R_{j}-1\right)-x\right)^{+}\right] \\
= & \alpha \sum_{k=0}^{\infty} \sigma^{k} \mathbf{E}^{\mathbb{Q}}\left[X_{j k} \mathbf{1}_{\left(R_{j} \geq \frac{\alpha+x}{\alpha}\right)}\right]-(\alpha+x) \sum_{k=0}^{\infty} \sigma^{k} \mathbf{E}^{\mathbb{Q}}\left[Y_{k} \mathbf{1}_{\left(R_{j} \geq \frac{\alpha+x}{\alpha}\right)}\right] .
\end{aligned}
$$

To derive $L_{0}, L_{1}$ we need to calculate $\mathbf{E}^{\mathbb{Q}}\left[X_{j k} \mathbf{1}_{\left(R_{j} \geq \frac{\alpha+x}{\alpha}\right)}\right]$ and $\mathbf{E}^{\mathbb{Q}}\left[Y_{k} \mathbf{1}_{\left(R_{j} \geq \frac{\alpha+x}{\alpha}\right)}\right]$ for $k=0,1$.

(a) Computation of $\mathbf{E}^{\mathbb{Q}}\left[X_{j 0} \mathbf{1}_{\left(R_{j} \geq \frac{\alpha+x}{\alpha}\right)}\right]$ and $\mathbf{E}^{\mathbb{Q}}\left[Y_{0} \mathbf{1}_{\left(R_{j} \geq \frac{\alpha+x}{\alpha}\right)}\right]$

Applying the equivalent inequality (24) for $R_{j} \geq \frac{\alpha+x}{\alpha}$ and ignoring the higher order terms in right-hand side of this inequality, we have 


$$
\begin{aligned}
& \mathbf{E}^{\mathbb{Q}}\left[X_{j 0} \mathbf{I}_{\left(R_{j} \geq \frac{\alpha+x}{\alpha}\right)}\right]=\mathbf{E}^{\mathbb{Q}}\left[C_{j} \mathrm{e}^{N_{j 0}} \mathbf{I}_{\left.\left(N_{j 0} \geq \Theta_{0}^{(j)}(x)+\sigma\left[N_{j 2} \Theta_{11}^{(j)}+\Theta_{12}^{(j)}(x)\right]+\sum_{i=2}^{\infty} \sigma^{i}\left[N_{j 2} \Theta_{i 1}^{(j)}+\Theta_{i 2}^{(j)}(x)\right]\right)\right]}\right. \\
= & \mathbf{E}^{\mathbb{Q}}\left[C_{j} \mathrm{e}^{N_{j 0}} \mathbf{I}_{\left(N_{j 0} \geq \Theta_{0}^{(j)}(x)+\sigma\left[N_{j 2} \Theta_{11}^{(j)}+\Theta_{12}^{(j)}(x)\right]\right)}\right]+o(\sigma),
\end{aligned}
$$

and

$$
\mathbf{E}^{\mathbb{Q}}\left[Y_{0} \mathbf{I}_{\left(R_{j} \geq \frac{\alpha+x}{\alpha}\right)}\right]=\mathbf{E}^{\mathbb{Q}}\left[Y_{0} \mathbf{I}_{\left(N_{j 0} \geq \Theta_{0}^{(j)}(x)+\sigma\left[N_{j 2} \Theta_{11}^{(j)}+\Theta_{12}^{(j)}(x)\right]\right)}\right]+o(\sigma) .
$$

Note that $C_{j}, \Theta_{0}^{(j)}(x), \Theta_{11}^{(j)}, \Theta_{12}^{(j)}(x)$ are deterministic functions, $N_{j 0}$ is a random variable with Gaussian distribution $N\left(0, \Sigma_{00}^{(j)}\right)$ and independent of standard Normal random variable $N_{j 2}$. Denote by $f_{\Sigma_{00}^{(j)}}(x)$ the density function of $N_{j 0}$ and $\Phi(x)$ the standard Normal distribution function. Taking conditional expectation with respect to $N_{j 2}$, we have

$$
\begin{aligned}
& \mathbf{E}^{\mathbb{Q}}\left[X_{j 0} \mathbf{I}_{\left(R_{j} \geq \frac{\alpha+x}{\alpha}\right)}\right]=C_{j} \mathbf{E}^{\mathbb{Q}}\left[\int_{\Theta_{0}^{(j)}(x)+\sigma\left[N_{j 2} \Theta_{11}^{(j)}+\Theta_{12}^{(j)}(x)\right]}^{\infty} \mathrm{e}^{x} f_{\Sigma_{00}^{(j)}}(x) d x\right]+o(\sigma) \\
= & C_{j} \mathrm{e}^{\frac{\Sigma_{00}^{(j)}}{2}} \mathbf{E}^{\mathbb{Q}}\left\{1-\Phi\left[\frac{\Theta_{0}^{(j)}(x)-\Sigma_{00}^{(j)}}{\sqrt{\Sigma_{00}^{(j)}}}+\sigma\left(\frac{N_{j 2} \Theta_{11}^{(j)}+\Theta_{12}^{(j)}(x)}{\sqrt{\Sigma_{00}^{(j)}}}\right)\right]\right\}+o(\sigma),
\end{aligned}
$$

and

$$
\mathbf{E}^{\mathbb{Q}}\left[Y_{0} \mathbf{I}_{\left(R_{j} \geq \frac{\alpha+x}{\alpha}\right)}\right]=Y_{0}-Y_{0} \mathbf{E}^{\mathbb{Q}}\left\{\Phi\left[H_{0}^{(j)}(x)+\sigma\left(\frac{N_{j 2} \Theta_{11}^{(j)}}{\sqrt{\Sigma_{00}^{(j)}}}+H_{12}^{(j)}(x)\right)\right]\right\}+o(\sigma),
$$

where

$$
G_{0}^{(j)}(x)=\frac{\Theta_{0}^{(j)}(x)-\Sigma_{00}^{(j)}}{\sqrt{\Sigma_{00}^{(j)}}}, \quad H_{0}^{(j)}(x)=\frac{\Theta_{0}^{(j)}(x)}{\sqrt{\Sigma_{00}^{(j)}}}, \quad \text { and } \quad H_{12}^{(j)}(x)=\frac{\Theta_{12}^{(j)}(x)}{\sqrt{\Sigma_{00}^{(j)}}}
$$

Again by Taylor's expansion of the distribution function $\Phi(y)$ around the points $y=G_{0}^{(j)}(x)$ and $y=H_{0}^{(j)}(x)$ respectively, we get

$$
\begin{aligned}
& \mathbf{E}^{\mathbb{Q}}\left[X_{j 0} \mathbf{I}_{\left(R_{j} \geq \frac{\alpha+x}{\alpha}\right)}\right]=C_{j} \mathrm{e}^{\frac{\Sigma_{00}^{(j)}}{2}} \mathbf{E}^{\mathbb{Q}}\left\{1-\sum_{n=0}^{\infty} \frac{D^{n} \Phi\left[G_{0}^{(j)}(x)\right]}{n !}\left[\sigma\left(\frac{N_{j 2} \Theta_{11}^{(j)}}{\sqrt{\Sigma_{00}^{(j)}}}+H_{12}^{(j)}(x)\right)\right]^{n}\right\}+o(\sigma) \\
= & C_{j} \mathrm{e}^{\frac{\Sigma_{00}^{(j)}}{2}}-C_{j} \mathrm{e}^{\frac{\Sigma_{00}^{(j)}}{2}} \Phi\left[G_{0}^{(j)}(x)\right]-\sigma C_{j} \mathrm{e}^{\frac{\Sigma_{00}^{(j)}}{2}} D^{1} \Phi\left[G_{0}^{(j)}(x)\right] H_{12}^{(j)}(x)+o(\sigma),
\end{aligned}
$$

and

$$
\begin{aligned}
& \mathbf{E}^{\mathbb{Q}}\left[Y_{0} \mathbf{I}_{\left(R_{j} \geq \frac{\alpha+x}{\alpha}\right)}\right]=Y_{0}-Y_{0} \mathbf{E}^{\mathbb{Q}}\left\{\sum_{n=0}^{\infty} \frac{D^{n} \Phi\left[H_{0}^{(j)}(x)\right]}{n !}\left[\sigma\left(\frac{N_{j 2} \Theta_{11}^{(j)}}{\sqrt{\Sigma_{00}^{(j)}}}+H_{12}^{(j)}(x)\right)\right]^{n}\right\}+o(\sigma) \\
= & Y_{0}-Y_{0} \Phi\left[H_{0}^{(j)}(x)\right]-\sigma Y_{0} D^{1} \Phi\left[H_{0}^{(j)}(x)\right] H_{12}^{(j)}(x)+o(\sigma),
\end{aligned}
$$

where $D^{n} \Phi(x), n=1,2, \cdots$ denote the $n$th derivative of $\Phi(x)$.

(b) Computation of $\mathbf{E}^{\mathbb{Q}}\left[X_{j 1} \mathbf{1}_{\left(R_{j} \geq \frac{\alpha+x}{\alpha}\right)}\right]$ and $\mathbf{E}^{\mathbb{Q}}\left[Y_{1} \mathbf{1}_{\left(R_{j} \geq \frac{\alpha+x}{\alpha}\right)}\right]$ 
In order to compute $\mathbf{E}^{\mathbb{Q}}\left[X_{j 1} \mathbf{1}_{\left(R_{j} \geq \frac{\alpha+x}{\alpha}\right)}\right]$ and $\mathbf{E}^{\mathbb{Q}}\left[Y_{1} \mathbf{1}_{\left(R_{j} \geq \frac{\alpha+x}{\alpha}\right)}\right]$, we introduce the notations $N_{j 3}, N_{j 4}, N_{j 5}$.

$$
\begin{aligned}
& N_{j 3}=\int_{0}^{j-1} A_{1}(t) d t=\int_{0}^{j-1} \int_{s}^{j-1} \mathrm{e}^{-k(t-s)} \sqrt{r^{(0)}(s)-\varphi(s)} d t d B_{2}(s), \\
& N_{j 4}=\int_{j}^{N} A_{1}(t) d t=\int_{0}^{j} \int_{j}^{N} \mathrm{e}^{-k(t-s)} \sqrt{r^{(0)}(s)-\varphi(s)} d t d B_{2}(s) \\
& +\int_{j}^{N} \int_{s}^{N} \mathrm{e}^{-k(t-s)} \sqrt{r^{(0)}(s)-\varphi(s)} d t d B_{2}(s), \\
& N_{j 5}=\int_{0}^{N} A_{1}(t) d t=\int_{0}^{N} \int_{s}^{N} \mathrm{e}^{-k(t-s)} \sqrt{r^{(0)}(s)-\varphi(s)} d t d B_{2}(s) .
\end{aligned}
$$

The variance of $N_{j i}, i=3,4,5$ is

$$
\begin{aligned}
\Sigma_{33}^{(j)} & =\operatorname{Var}\left[N_{j 3}\right]=\int_{0}^{j-1}\left(\int_{s}^{j-1} \mathrm{e}^{-k(t-s)} \sqrt{r^{(0)}(s)-\varphi(s)} d t\right)^{2} d s \\
\Sigma_{44}^{(j)} & =\operatorname{Var}\left[N_{j 4}\right] \\
& =\int_{0}^{j}\left(\int_{j}^{N} \mathrm{e}^{-k(t-s)} \sqrt{r^{(0)}(s)-\varphi(s)} d t\right)^{2} d s+\int_{j}^{N}\left(\int_{s}^{N} \mathrm{e}^{-k(t-s)} \sqrt{r^{(0)}(s)-\varphi(s)} d t\right)^{2} d s \\
\Sigma_{55}^{(j)}= & \operatorname{Var}\left[N_{j 5}\right]=\int_{0}^{N}\left(\int_{s}^{N} \mathrm{e}^{-k(t-s)} \sqrt{r^{(0)}(s)-\varphi(s)} d t\right)^{2} d s
\end{aligned}
$$

and the covariances between $N_{j 0}$ and $N_{j i}, i=3,4,5$, respectively, are

$$
\begin{aligned}
& \Sigma_{03}^{(j)}=\operatorname{Cov}\left(N_{j 0}, N_{j 3}\right)=0, \\
& \Sigma_{04}^{(j)}=\operatorname{Cov}\left(N_{j 0}, N_{j 4}\right)=\sigma_{S} \rho \int_{j-1}^{j} \int_{j}^{N} \mathrm{e}^{-k(t-s)} \sqrt{r^{(0)}(s)-\varphi(s)} d t d s, \\
& \Sigma_{05}^{(j)}=\operatorname{Cov}\left(N_{j 0}, N_{j 5}\right)=\sigma_{S} \rho \int_{j-1}^{j} \int_{s}^{N} \mathrm{e}^{-k(t-s)} \sqrt{r^{(0)}(s)-\varphi(s)} d t d s .
\end{aligned}
$$

Note that for $i=3,4,5, N_{j i}$ follows a Gaussian distribution. By Gaussian distribution properties, we have under the condition $N_{j 0}=x$, the conditional distribution of $N_{j i}, N_{j i}=\frac{\Sigma_{0 i}^{(j)}}{\Sigma_{00}^{(j)}} x+$ $N_{j} \sqrt{\left|\Sigma_{i i}^{(j)}-\frac{\Sigma_{0 i}^{(j)} \Sigma_{0 i}^{(j)}}{\Sigma_{00}^{(j)}}\right|}=a_{i}^{(j)} x+N_{j} b_{i}^{(j)}$, where $N_{j} \sim N(0,1)$ and independent on $N_{j 0}$ and $a_{i}^{(j)}=$ $\Sigma_{0 i}^{(j)} / \Sigma_{00}^{(j)}, b_{i}^{(j)}=\sqrt{\left|\Sigma_{i i}^{(j)}-\frac{\Sigma_{0 i}^{(j)} \Sigma_{0 i}^{(j)}}{\Sigma_{00}^{(j)}}\right|}$.

By definition of $X_{j 1}$ and $Y_{1}$, then

$$
X_{j 1}=-X_{j 0} \int_{(0, j-1) \cup(j, N)} A_{1}(t) d t=-C_{j} \mathrm{e}^{N_{j 0}}\left(N_{j 3}+N_{j 4}\right) \quad \text { and } \quad Y_{1}=-Y_{0} \int_{0}^{N} A_{1}(t) d t=-Y_{0} N_{j 5} .
$$

Recalling the argument about relation between $N_{j 0}$ and $N_{j 3}, N_{j 4}$ and $N_{j 5}$, and using the equivalent 
inequality of $R_{j} \geq \frac{\alpha+x}{\alpha}$, we have

$$
\begin{aligned}
& \mathbf{E}^{\mathbb{Q}}\left[X_{j 1} \mathbf{I}_{\left(R_{j} \geq \frac{\alpha+x}{\alpha}\right)}\right] \\
= & \mathbf{E}^{\mathbb{Q}}\left[-C_{j} \mathrm{e}^{N_{j 0}} N_{j 3} \mathbf{I}_{\left.\left(N_{j 0} \geq \Theta_{0}^{(j)}(x)+\sigma\left[N_{j 2} \Theta_{11}^{(j)}+\Theta_{12}^{(j)}(x)\right]\right)\right]}\right] \\
& +\mathbf{E}^{\mathbb{Q}}\left[-C_{j} \mathrm{e}^{N_{j 0}} N_{j 4} \mathbf{I}_{\left.\left(N_{j 0} \geq \Theta_{0}^{(j)}(x)+\sigma\left[N_{j 2} \Theta_{11}^{(j)}+\Theta_{12}^{(j)}(x)\right]\right)\right]+o(\sigma)}\right. \\
= & -C_{j} \mathbf{E}^{\mathbb{Q}}\left[\int_{\Theta_{0}^{(j)}(x)+\sigma\left[N_{j 2} \Theta_{11}^{(j)}+\Theta_{12}^{(j)}(x)\right]}^{\infty} \mathrm{e}^{x}\left[a_{3}^{(j)} x+N_{j} b_{3}^{(j)}\right] f_{\Sigma_{00}^{(j)}}(x) d x\right] \\
& -C_{j} \mathbf{E}^{\mathbb{Q}}\left[\int_{\Theta_{0}^{(j)}(x)+\sigma\left[N_{j 2} \Theta_{11}^{(j)}+\Theta_{12}^{(j)}(x)\right]}^{\infty} \mathrm{e}^{x}\left[a_{4}^{(j)} x+N_{j} b_{4}^{(j)}\right] f_{\Sigma_{00}^{(j)}}(x) d x\right]+o(\sigma),
\end{aligned}
$$

and

$$
\begin{aligned}
& \mathbf{E}^{\mathbb{Q}}\left[Y_{1} \mathbf{I}_{\left(R_{j} \geq \frac{\alpha+x}{\alpha}\right)}\right]=\mathbf{E}^{\mathbb{Q}}\left[-Y_{0} N_{j 5} \mathbf{I}_{\left(N_{j 0} \geq \Theta_{0}^{(j)}(x)+\sigma\left[N_{j 2} \Theta_{11}^{(j)}+\Theta_{12}^{(j)}(x)\right]\right)}\right]+o(\sigma) \\
= & -Y_{0} \mathbf{E}^{\mathbb{Q}}\left[\int_{\Theta_{0}^{(j)}(x)+\sigma\left[N_{j 2} \Theta_{11}^{(j)}+\Theta_{12}^{(j)}(x)\right]}^{\infty}\left[a_{5}^{(j)} x+N_{j} b_{5}^{(j)}\right] f_{\Sigma_{00}^{(j)}}(x) d x\right]+o(\sigma) .
\end{aligned}
$$

Since $N_{j}$ is independent of $N_{j i}$ for $i=0,1, \cdots, 5$ and its expectation is 0 , then

$$
\begin{aligned}
& \mathbf{E}^{\mathbb{Q}}\left[X_{j 1} \mathbf{I}_{\left(R_{j} \geq \frac{\alpha+x}{\alpha}\right)}\right]=-C_{j}\left[a_{3}^{(j)}+a_{4}^{(j)}\right] \mathbf{E}^{\mathbb{Q}}\left[\int_{\Theta_{0}^{(j)}(x)+\sigma\left[N_{j 2} \Theta_{11}^{(j)}+\Theta_{12}^{(j)}(x)\right]}^{\infty} \mathrm{e}^{x} x f_{\Sigma_{00}^{(j)}}(x) d x\right]+o(\sigma) \\
= & -C_{j}\left[a_{3}^{(j)}+a_{4}^{(j)}\right] \sqrt{\frac{\Sigma_{00}^{(j)}}{2 \pi}} \mathrm{e}^{\frac{\Sigma_{00}^{(j)}}{2}} \mathbf{E}^{\mathbb{Q}}\left[\exp \left(-\frac{\left.\left[\Theta_{0}^{(j)}(x)-\Sigma_{00}^{(j)}+\sigma\left(N_{j 2} \Theta_{11}^{(j)}+\Theta_{12}^{(j)}(x)\right)\right]^{2}\right)}{2 \Sigma_{00}^{(j)}}\right)\right] \\
& +C_{j}\left[a_{3}^{(j)}+a_{4}^{(j)}\right] \Sigma_{00}^{(j)} \mathrm{e}^{\frac{\Sigma_{00}^{(j)}}{2}} \mathbf{E}^{\mathbb{Q}}\left\{\Phi\left[G_{0}^{(j)}(x)+\sigma\left(\frac{N_{j 2} \Theta_{i 1}^{(j)}}{\sqrt{\Sigma_{00}^{(j)}}+H_{i 2}^{(j)}(x)}\right)\right]-1\right\}+o(\sigma),
\end{aligned}
$$

and

$$
\begin{aligned}
& \mathbf{E}^{\mathbb{Q}}\left[Y_{1} \mathbf{I}_{\left(R_{j} \geq \frac{\alpha+x}{\alpha}\right)}\right]=-Y_{0} a_{5}^{(j)} \mathbf{E}^{\mathbb{Q}}\left[\int_{\Theta_{0}^{(j)}(x)+\sigma\left[N_{j 2} \Theta_{11}^{(j)}+\Theta_{12}^{(j)}(x)\right]}^{\infty} x f_{\Sigma_{00}^{(j)}}(x) d x\right]+o(\sigma) \\
= & -Y_{0} a_{5}^{(j)} \sqrt{\frac{\Sigma_{00}^{(j)}}{2 \pi}} \mathbf{E}^{\mathbb{Q}}\left[\exp \left(-\frac{\left[\Theta_{0}^{(j)}(x)+\sigma\left(N_{j 2} \Theta_{11}^{(j)}+\Theta_{12}^{(j)}(x)\right)\right]^{2}}{2 \Sigma_{00}^{(j)}}\right)\right]+o(\sigma) .
\end{aligned}
$$

Applying Taylor's expansion to the function $\mathrm{e}^{-y}$ around $y=\frac{\left(\Theta_{0}^{(j)}(x)-\Sigma_{00}^{(j)}\right)^{2}}{2 \Sigma_{00}^{(j)}}$ and $y=\frac{\left(\Theta_{0}^{(j)}(x)\right)^{2}}{2 \Sigma_{00}^{(j)}}$, 
and also to the function $\Phi(y)$ around the point $y=G_{0}^{(j)}(x)$, we have

$$
\begin{aligned}
& \mathbf{E}^{\mathbb{Q}}\left[X_{j 1} \mathbf{I}_{\left(R_{j} \geq \frac{\alpha+x}{\alpha}\right)}\right] \\
& =-C_{j}\left[a_{3}^{(j)}+a_{4}^{(j)}\right] \sqrt{\frac{\Sigma_{00}^{(j)}}{2 \pi}} \mathrm{e}^{\frac{\Sigma_{00}^{(j)}}{2}-\frac{\left(\Theta_{0}^{(j)}(x)-\Sigma_{00}^{(j)}\right)^{2}}{2 \Sigma_{00}^{(j)}}}\left[1-\sigma G_{0}^{(j)}(x) H_{12}^{(j)}(x)+o(\sigma)\right] \\
& +C_{j}\left[a_{3}^{(j)}+a_{4}^{(j)}\right] \Sigma_{00}^{(j)} \mathrm{e}^{\frac{\Sigma_{00}^{(j)}}{2}}\left\{\Phi\left[G_{0}^{(j)}(x)\right]+\sigma D^{1} \Phi\left[G_{0}^{(j)}(x)\right] H_{12}^{(j)}(x)+o(\sigma)-1\right\}+o(\sigma) \\
& =C_{j}\left[a_{3}^{(j)}+a_{4}^{(j)}\right] \mathrm{e}^{\frac{\Sigma_{00}^{(j)}}{2}}\left\{\Sigma_{00}^{(j)} \Phi\left[G_{0}^{(j)}(x)\right]-\sqrt{\frac{\Sigma_{00}^{(j)}}{2 \pi}} \mathrm{e}^{-\frac{\left(\Theta_{0}^{(j)}(x)-\Sigma_{00}^{(j)}\right)^{2}}{2 \Sigma_{00}^{(j)}}}-\Sigma_{00}^{(j)}\right\} \\
& +\sigma C_{j}\left[a_{3}^{(j)}+a_{4}^{(j)}\right] \mathrm{e}^{\frac{\Sigma_{00}^{(j)}}{2}}\left\{\Sigma_{00}^{(j)} D^{1} \Phi\left[G_{0}^{(j)}(x)\right] H_{12}^{(j)}(x)+\sqrt{\frac{\Sigma_{00}^{(j)}}{2 \pi}} \mathrm{e}^{-\frac{\left(\Theta_{0}^{(j)}(x)-\Sigma_{00}^{(j)}\right)^{2}}{2 \Sigma_{00}^{(j)}}} G_{0}^{(j)}(x) H_{12}^{(j)}(x)\right\}+o(\sigma),
\end{aligned}
$$

and

$$
\begin{aligned}
& \mathbf{E}^{\mathbb{Q}}\left[Y_{1} \mathbf{I}_{\left(R_{j} \geq \frac{\alpha+x}{\alpha}\right)}\right]=-Y_{0} a_{5}^{(j)} \sqrt{\frac{\Sigma_{00}^{(j)}}{2 \pi}} \mathbf{E}^{\mathbb{Q}}\left[\exp \left(-\frac{\left[\Theta_{0}^{(j)}(x)+\sigma\left(N_{j 2} \Theta_{11}^{(j)}+\Theta_{12}^{(j)}(x)\right)\right]^{2}}{2 \Sigma_{00}^{(j)}}\right)\right]+o(\sigma) \\
= & -Y_{0} a_{5}^{(j)} \sqrt{\frac{\Sigma_{00}^{(j)}}{2 \pi}} \mathrm{e}^{-\frac{\Theta_{0}^{(j)}(x)^{2}}{2 \Sigma_{00}^{(j)}}}\left[1-\sigma H_{0}^{(j)}(x) H_{12}^{(j)}(x)\right]+o(\sigma) .
\end{aligned}
$$

To end up the computation of $\mathbf{E}^{\mathbb{Q}}\left[\mathrm{e}^{-\int_{0}^{N} r(s) d s}\left(\alpha\left(R_{j}-1\right)-x\right)^{+}\right]$, we just need to apply formulas (29), (30), (33) and (34) into (28). So that

$$
\begin{aligned}
& \mathbf{E}^{\mathbb{Q}}\left[\mathrm{e}^{-\int_{0}^{N} r(s) d s}\left(\alpha\left(R_{j}-1\right)-x\right)^{+}\right] \\
= & \alpha \mathbf{E}^{\mathbb{Q}}\left[X_{j 0} \mathbf{1}_{\left(R_{j} \geq \frac{\alpha+x}{\alpha}\right)}\right]-(\alpha+x) \mathbf{E}^{\mathbb{Q}}\left[Y_{0} \mathbf{1}_{\left(R_{j} \geq \frac{\alpha+x}{\alpha}\right)}\right] \\
& +\sigma\left\{\alpha \mathbf{E}^{\mathbb{Q}}\left[X_{j 1} \mathbf{1}_{\left(R_{j} \geq \frac{\alpha+x}{\alpha}\right)}\right]-(\alpha+x) \sigma \mathbf{E}^{\mathbb{Q}}\left[Y_{1} \mathbf{1}_{\left(R_{j} \geq \frac{\alpha+x}{\alpha}\right)}\right]\right\}+o(\sigma) \\
= & M_{0}(j, x)+\sigma M_{1}(j, x)+o(\sigma),
\end{aligned}
$$

where

$$
\begin{aligned}
M_{0}(j, x)= & \alpha C_{j} \mathrm{e}^{\frac{\Sigma_{00}^{(j)}}{2}}\left(1-\Phi\left[G_{0}^{(j)}(x)\right]\right)-(\alpha+x) Y_{0}\left(1-\Phi\left[H_{0}^{(j)}(x)\right]\right) \\
M_{1}(j, x)= & \alpha C_{j} \mathrm{e}^{\frac{\Sigma_{00}^{(j)}}{2}}\left[\left(a_{3}^{(j)}+a_{4}^{(j)}\right)\left(\Sigma_{00}^{(j)} \Phi\left[G_{0}^{(j)}(x)\right]-\sqrt{\frac{\Sigma_{00}^{(j)}}{2 \pi}} \mathrm{e}^{-\frac{\left(\Theta_{0}^{(j)}(x)-\Sigma_{00}^{(j)}\right)^{2}}{2 \Sigma_{00}^{(j)}}}-\Sigma_{00}^{(j)}\right)\right. \\
& \left.-D^{1} \Phi\left[G_{0}^{(j)}(x)\right] H_{12}^{(j)}(x)\right]+(\alpha+x) Y_{0}\left[a_{5}^{(j)} \sqrt{\frac{\Sigma_{00}^{(j)}}{2 \pi}} \mathrm{e}^{-\frac{\Theta_{0}^{(j)}(x)^{2}}{2 \Sigma_{00}^{(j)}}}+D^{1} \Phi\left[H_{0}^{(j)}(x)\right] H_{12}^{(j)}(x)\right]
\end{aligned}
$$


Finally from (7) we can conclude that

$$
\begin{aligned}
L_{0} & =(1+N F) Y_{0}+\sum_{j=1}^{N}\left[M_{0}(j, F)-M_{0}(j, C)\right] \\
L_{1} & =\sum_{j=1}^{N}\left[M_{1}(j, F)-M_{1}(j, C)\right] .
\end{aligned}
$$

\section{References}

[1] Alfonsi, A. 2010. High order discretization schemes for the CIR process: Application to affine term structure and Heston models. Mathematics of Computation Vol. 79, No. 269, 209-237.

[2] Amin, K., Khanna, A. 1994. Convergence of American Option Values From Discrete-to Continuous-Time Financial Models. Mathematical Finance 4, 4, 289-304.

[3] Brigo, D., Mercurio, F. 2006. Interest Rate Models-Theory and Practice. Springer, Berlin.

[4] Costabile M, Gaudenzi, M., I.Massabò, Zanette, A. 2009. Evaluating fair premiums of equitylinked policies with surrender option in a bivariate model. Insurance: Mathematics and Economics 41, 317-338.

[5] Cox, J.C., Ingersoll, J., Ross, S. 1985. A Theory of the Term Structure of Interest Rates. Econometrica 53, 385-407

[6] Ethier, S.N., Kurtz, T. 1986. Markov Processes: Characterization and Convergence. John Wiley \& Sons, New York.

[7] Gaudenzi, M., Lepellere, M.A., Zanette, A. 2010. The Singular Point Method for Pricing PathDependent Options. Journal of Computational Finance 14-1, 29-56.

[8] Gerber, H., Shiu, E. 2003. Pricing lookback options and dynamic guarantees. North American Actuarial Journal 7 (1), 48-67.

[9] Hardy M.R. 2003. Investment Guarantees: Modelling and Risk Management for Equity-Linked Life Insurance. Wiley, New York.

[10] Hull J., White A. 1994. Numerical procedures for implementing term structure models I. Journal of Derivatives 2(1), 7-16.

[11] Kijima M., Wong T. 2007. Pricing of Ratchet equity-indexed annuities under stochastic interest rate. Insurance: Mathematics and Economics 41, 317-338.

[12] Kim Y., Kunitomo, N. 1999. Pricing options under stochastic interest rates: a new approach. Asia-Pacific Financial Markets (Kluwer) 6, 49-70.

[13] Kunitomo, N., Takahashi, A. 2003. On validity of the asymptotic expansion approach in contingent claim analysis. Annals of Applied Probability 13(3), 914-952.

[14] Kushner H. Dupuis P.G. 1992. Numerical Methods for Stochastic Control Problems in Continous Time. Springer Verlag, Berlin and New York.

[15] Lin, X.D., Tan, K.S. 2003. Valuation of equity-indexed annuities under stochastic interest rates. North American Auctuarial Journal 6, 72-91.

$\mathrm{RR}^{\circ} 8034$ 
[16] Nelson, D. B., Ramaswamy, K. 1990. Simple Binomial Processes as Diffusion Approximations in Financial Models. The Review of Financial Studies 3, 393-430

[17] Palmer, B.A. 2006. Equity-Indexed Annuities: Fundamental concepts and issues, Report of Insurance Information Institute, available at http://hbwinc.com/static/pdf/EIA-Booklet.pdf.

[18] Wei, J., Z. 1996. Valuing American Equity Options with a Stochastic Interest Rate: A Note. The Journal of Financial Engineering 2, 2, 195-206

[19] Wilmott P. 2002. Cliquet Options and Volatility Models. Wilmott Magazine 3, 78-83.

[20] 2009 Annuity Fact Book: A guide to information, trends, and data in the annuity industry. Insured Retirement Institute. 


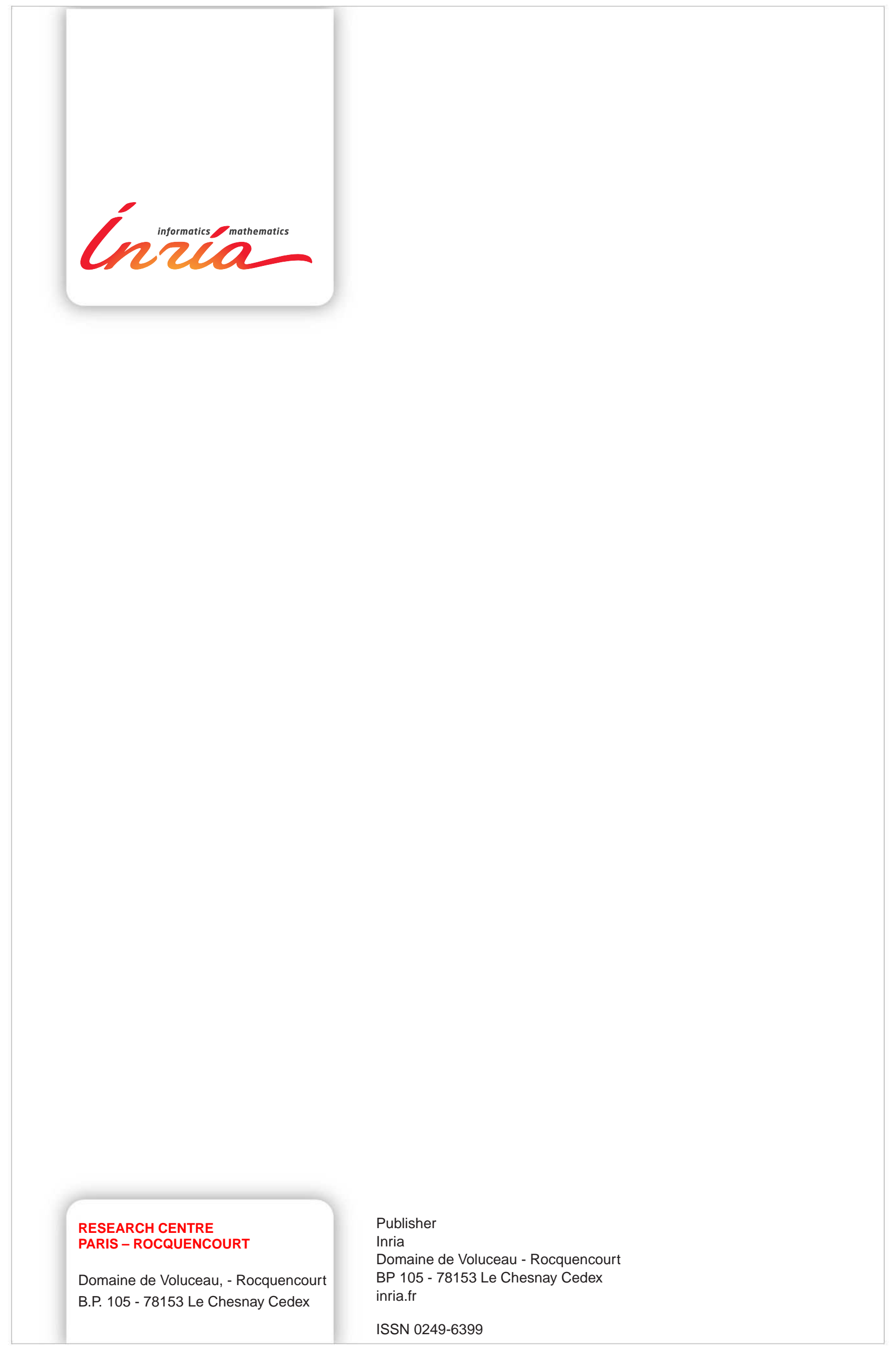

